\title{
MEASURES AND TENSORS II
}

\author{
JESƯS GIL DE LAMADRID
}

1. Introduction. The present work is a sequel to our previous article (10) with the same title. The major theme remains the study of the relationship between tensor products of spaces of functions and vector-valued measures on a space $S$. In (10) $S$ was a compact Hausdorff space. Here we extend our considerations to locally compact Hausdorff spaces. $\mathbf{B}(S)$ still stands for the Borel class of $S$.

Three types of vector-valued measures $m: \mathbf{B}(S) \rightarrow E, E$ a Banach space, are considered here $(\$ 3)$, namely, weak*, weak, and strong vector measures, but the concepts of weak and strong measures coincide. This result is due to Bartle, Dunford, and Schwartz (1) for abstract vector measures, i.e. defined on an abstract $\sigma$-algebra of sets. Their result shows that weak countable additivity implies strong countable additivity. Here we complete this result in our topological setting by showing (Lemma 3.1) that weak regularity implies strong regularity. By contrast, weak* vector measures $m: \mathbf{B}(S) \rightarrow E^{\prime}$ form a distinct class from strong $E^{\prime}$-valued measures on $S$. Here $E^{\prime}$ denotes the dual (conjugate) space of $E$. An example to illustrate this discrepancy is given in $\S 3$; but the most interesting examples of the same phenomenon are resolutions of the identity for certain spectral operators (in the sense of Dunford (7)). These last examples are discussed in $\$ 6$, the last section of this article and the one devoted to the discussion of questions that are best handled with the aid of the theory of tensor products. In $\S 3$ we also discuss Pettis (18) integrable mappings $f: S \rightarrow E$ with respect to a scalar-valued measure $\rho$. Each such $f$ has a natural norm $\|f\|$ and they all form a normed space that, when completed, yields a Banach space $W(\rho, E)$ which can be identified (Theorem 2.1) with a space of strong measures $m: \mathbf{B}(S) \rightarrow E$. Not all these measures can be identified with Pettis integrable mappings (an open question); but in Theorem 3.2 we give a condition on $m$ sufficient for such an identification to be possible. That theorem is a type of Lebesgue bounded convergence theorem.

Section 2 is devoted to adapting to the topological situation certain lemmas in the abstract theory of Bartle, Dunford, and Schwartz (1), which are needed here. We also present some auxiliary result designed to implement the adaptation of earlier work on compact spaces (10) and abstract $\sigma$-algebras (1) to the setting of locally compact spaces and the resulting $\sigma$-rings. This involves the

Received June 22, 1965. Part of this material was presented to the American Mathematical Society on November 23, 1962. Support of this research through National Science Foundation research grants (NSF G19752 and G 2560) is gratefully acknowledged. 
study of the natural extension (Lemma 2.1) of a finite scalar measure on a locally compact space $S$ to its one-point compactification.

In $\$ 4$ we show that the distinction between weak* measures $m: \mathbf{B}(S) \rightarrow E^{\prime}$ and strong measures $m: \mathbf{B}(S) \rightarrow E^{\prime}$ disappears in the presence of the additional assumption that $m$ has finite variation in the sense of Dinculeanu (2). This fact is used to fill a gap in the proof of a theorem of Ivan Singer (20). It also reveals the fact that under the assumption of finite variation the fact that the Banach space $E^{\prime}$ is the dual of another space is irrelevant and enables us to speak without ambiguity of vector measures $m: \mathbf{B}(S) \rightarrow E$ of finite variation, without the qualifying terms weak* or strong.

Vector measures $m$ of finite variation played a fundamental role in our work in (10) and their study is resumed here in $\$ \$ 4$ (see the previous paragraph), 5 , and 6 . The main problem considered in $\$ \$ 5$ and 6 is the following. Since a vector measure $m: \mathbf{B}(S) \rightarrow E$ of finite variation is absolutely continuous with respect to its scalar variation measure $\mu^{m}$ (i.e., $A \in \mathbf{B}(S)$ and $\mu^{m}(A)=0$ imply $m(A)=0$ ), one might wonder if there exists a Radon-Nikodym representation of $m$ in terms of $\mu^{m}$; namely, if there exists a mapping $h_{m}: S \rightarrow E$ such that, for $A \in \mathbf{B}(S)$,

$$
m(A)=\int_{A} h_{m}(s) d \mu^{m}(s) .
$$

If we insist that $h_{m}$ be Bochner integrable with respect to $\mu^{m}$, the answer can be given in terms of tensor products. The isometric isomorphism represented by the following inclusion was established in (10) for compact $S$ and is extended here to locally compact spaces (Theorem 6.2):

$$
C^{\prime}(S) \otimes_{\gamma} E \subset M(S, E) .
$$

In (1.2) $C^{\prime}(S)$ is the dual space of the space of all scalar-valued continuous functions on $S$ vanishing at $\infty$, and $M(S, E)$ the Banach space of all vector measures $m: \mathbf{B}(S) \rightarrow E$ of finite variation with the total variation norm. Now the measures $m$ having a representation (1.1) with $h_{m}$ Bochner integrable are characterized (Theorem 6.3) as those elements of $M(S, E)$ that belong to $C^{\prime}(S) \otimes_{\gamma} E$, in the context of (1.2). Using this theorem, Phillips spaces (discussed in (10)) are characterized (Theorem 6.4) as those spaces $E$ for which the inclusion in (1.2) is actually equality for every locally compact Hausdorff space $S$. These results, in turn, are based on some preliminary results developed in $\$ 5$.

For the special case in which $E$ is a space of bounded linear transformations, a representation (1.1) has already been given by Dinculeanu and Foias (5) for all $m: \mathbf{B}(S) \rightarrow E$, where $h_{m}$ is, of course, not always Bochner integrable, but has weaker measurability properties. It turns out that a very simple proof of this result is made possible by the use of tensor products, which permits the elimination of a separability assumption made in (5). That proof is given here (Theorem 6.5). 
In addition to (1.2) the following tensor relation is given in $\$ 6$ (Theorem $6.1)$ :

$$
L_{1}(\rho) \otimes_{\lambda} E=W(\rho, E) .
$$

This is, in a way, a parallel result to the relation

$$
L_{1}(\rho) \otimes_{\gamma} E=L_{1}(\rho, E)
$$

of Grothendieck (12).

2. Scalar measures. We shall be dealing with notions that are defined in terms of either the field of real numbers or the field of complex numbers. All considerations contained in the present article, except for those referring specifically to real numbers (e.g., $\epsilon>0$ ) are valid, whether one uses systematically throughout one field of scalars or the other. Therefore we prefer to leave the identity of the specific field ambiguous and shall follow the convention, initiated in (10), of referring to it simply as the field of scalars. The term Banach space and other functional-analytic terms should be interpreted in this spirit. The letter $S$ will always denote a locally compact Hausdorff space, $\mathbf{B}(S)$ its Borel class. In dealing with measures on $S$, we shall follow, on the whole, the conventions and notations set down by Halmos (14). We emphasize the following exception. In most of this work, we need only concern ourselves with finite regular measures. Consequently the term scalar measure, without any further qualifications, shall mean a countably additive regular scalarvalued (hence bounded) function on $\mathbf{B}(S)$. In a few places the assumption of finiteness may be dropped (e.g., in connection with the Pettis integral in §3). This is emphasized by referring, in those situations, to not-necessarily-finite (but still regular) non-negative measures. In other places it is necessary to consider abstract measures, and they will be designated as such. These are measures defined on abstract $\sigma$-rings, not naturally connected with a topological space.

The symbol $E$ will denote a Banach space and $E^{\prime}$ its dual (conjugate) space. For $x \in E$ and $x^{\prime} \in E^{\prime}$, the symbol $\left\langle x, x^{\prime}\right\rangle$ denotes the action of $x$ and $x^{\prime}$ on each other. The space of all continuous scalar-valued functions defined on $S$, vanishing at $\infty$, will be denoted by $C(S)$. This space is a Banach space under the sup norm \|\|$_{\infty}$. The dual space $C^{\prime}(S)$ of $C(S)$ is (17, Theorem 10, p. 1012) the space of all scalar-valued measures $\mu$ on $S$. The dual norm is the total variation, denoted by $\bar{\mu}(S)$. More generally, the variation measure of $\mu$ is a non-negative real-valued measure, denoted by $\bar{\mu}$. We shall use the symbol $\mu$, with deliberate ambiguity, to denote a measure and the functional it defines. Thus, the use of the symbols $\langle\phi, \mu\rangle$ for $\phi \in C(S)$ and $\mu(A)$ for $A \in \mathbf{B}(S)$ should cause no confusion.

The purpose of this section is to present some preliminary results on scalar measures. Some of them may be well known, but the author has been unable 
to find adequate references for them. Let $S_{\infty}$ denote the one-point compactification of $S$. We know that $C(S)$ can be imbedded isometrically in $C\left(S_{\infty}\right)$ in a natural way. We refer to this imbedding as the canonical imbedding of $C(S)$ in $C\left(S_{\infty}\right)$. After the canonical imbedding is performed, the functions of $C(S)$ become functions defined on $S_{\infty}$ which actually vanish at $\infty$. We denote the canonical imbedding by $C(S) \subset C\left(S_{\infty}\right)$. We also know that $C(S)$ is actually a hyperplane of $C\left(S_{\infty}\right)$, which is the null space of the unit point measure $\delta_{\infty}$ concentrated at $\infty$. We shall often have occasion to use the decomposition of a function $\psi \in C\left(S_{\infty}\right)$ given by

$$
\psi=\left(\psi-\kappa_{\psi}\right)+\kappa_{\psi}
$$

where $\kappa_{\psi}$ is the constant function whose value coincides with $\psi(\infty)$. This decomposition is, of course, the decomposition of $\psi$ according to the hyperplane $C(S)$ and the functional $\delta_{\infty}$. We have already used the notation $\bar{\mu}(S)$ to denote the total variation of a measure $\mu$. The symbol $\mu(S)$ may also be defined easily, even if $S$ is not measurable and $\mu$ is not a non-negative measure. Since, as we have seen, $\mu(S)$ is well defined for non-negative measures, it suffices to express a general measure $\mu$ in terms of non-negative measures (Jordan decomposition, etc.) and to express $\mu(S)$ in terms of its corresponding values for the nonnegative measures that give $\mu$.

Lemma 2.1. The dual space $C^{\prime}(S)$ of $C(S)$ can be imbedded isometrically in the space $C^{\prime}\left(S_{\infty}\right)$ as a closed hyperplane of $C^{\prime}\left(S_{\infty}\right)$ in such a way that the image $\mu_{\infty}$, under this imbedding, of a measure $\mu$ of $C^{\prime}(S)$ is an extension of $\mu$ from $\mathbf{B}(S)$ to $\mathbf{B}\left(S_{\infty}\right)$.

Proof. Let us consider $C(S)$ as a subset of $C\left(S_{\infty}\right)$, as indicated above. Let $\mu \in C^{\prime}(S)$. Let us extend the functional $\mu$ on $C(S)$ to a functional $\mu_{\infty}$ on $C\left(S_{\infty}\right)$. For $\psi \in C\left(S_{\infty}\right)$, with decomposition $(2.1)$, we define $\left\langle\psi, \mu_{\infty}\right\rangle$ as

$$
\left\langle\psi, \mu_{\infty}\right\rangle=\left\langle\psi-\kappa_{\psi}, \mu\right\rangle+\mu(S) \psi(\infty) .
$$

Clearly the functional $\mu_{\infty}$ extends the functional $\mu$ and is non-negative when $\mu$ is non-negative. This easily implies, for non-negative $\mu$, that the measure $\mu_{\infty}$ extends the measure $\mu$. For general scalar $\mu$, we express it in terms of nonnegative measures, and verify, in the general case, that the measure $\mu_{\infty}$ defined on $\mathbf{B}\left(S_{\infty}\right)$ extends the measure $\mu$ defined on $\mathbf{B}(S)$. A similar argument yields the fact that the imbedding $\mu \rightarrow \mu_{\infty}$ is isometric. Clearly $\mu_{\infty}$ is concentrated on $S$, i.e., $\mu_{\infty}(\{\infty\})=0$. We can also show, conversely, that a measure $\nu$ on $S_{\infty}$ is of the form $\mu_{\infty}$, for $\mu \in C^{\prime}(S)$, if it is concentrated on $S$. We now have for any $\nu \in C^{\prime}\left(S_{\infty}\right)$, the decomposition

$$
\nu=\nu-\nu(\{\infty\}) \delta_{\infty}+\nu(\{\infty\}) \delta_{\infty},
$$

where $\nu-\nu(\{\infty\}) \delta_{\infty}$ clearly belongs to $C^{\prime}(S) \subset C^{\prime}\left(S_{\infty}\right)$. It follows from (2.3) that $C^{\prime}(S)$ is indeed a closed hyperplane of $C^{\prime}\left(S_{\infty}\right)$. This completes our proof. 
In the proof of Lemma 2.1 we have established the extensibility of a measure $\mu$ on $S$ to $\mu_{\infty}$ on $S_{\infty}$ by means of the extensibility of the corresponding functional. A more direct approach, which we now sketch, will be quite useful later on in various places, and has the added advantage of being valid for finite abstract Borel measures (i.e. finite, not necessarily regular Borel measures) on $S$. Clearly, we can limit ourselves to non-negative $\mu$. Even if $S$ is not measurable (that is, not contained in $\mathbf{B}(S)$ ), there exists a set $P \in \mathbf{B}(S)$, on which $\mu$ is concentrated. This is because there is a sequence $C_{1} \subset C_{2} \subset \ldots$ of compact sets such that $\lim _{i} \mu\left(C_{i}\right)=\mu(S)$. We merely take $P$ to be the union of these compact sets. One can show easily that for any $B \in \mathbf{B}\left(S_{\infty}\right), B \cap P \in \mathbf{B}(S)$. We now define $\mu_{\infty}(B)$ as $\mu(B \cap P)$.

We summarize this discussion in the following lemma for the purpose of future reference.

Lemma 2.2. Every finite abstract (not necessarily regular) scalar-valued Borel measure on $S$ can be extended to a unique abstract Borel measure on $S_{\infty}$, concentrated on $S$ with the same total variation as the original measure on $S$.

We now examine a single non-negative measure $\mu \in C^{\prime}(S)$ and its extension $\mu_{\infty} \in C^{\prime}\left(S_{\infty}\right)$. We shall show that $L_{1}(\mu)$ can be identified with $L_{1}\left(\mu_{\infty}\right)$. To simplify our notation, we shall use the symbol \|\|$_{1}$ to denote the natural norm of both of these spaces. The arguments that are soon to follow will show that no confusion can possibly result.

Lemma 2.3. The natural imbedding $C(S) \subset C\left(S_{\infty}\right)$ can be extended to a unique isometric isomorphism represented by the equation

$$
L_{1}(\mu)=L_{1}\left(\mu_{\infty}\right) .
$$

Proof. It is clear that the natural imbedding $C(S) \subset C\left(S_{\infty}\right)$ preserves not only the norm \|\|$_{\infty}$ but also the norm \|\|$_{1}$. Consequently, since $C(S)$ is dense in $L_{1}(\mu)$, it follows that the natural imbedding can be extended to an isometric imbedding $L_{1}(\mu) \subset L_{1}\left(\mu_{\infty}\right)$. It simply remains to show that this imbedding is actually onto. Since $C\left(S_{\infty}\right)$ is dense in $L_{1}\left(S_{\infty}\right)$, it suffices to show that every $\psi \in C\left(S_{\infty}\right)$ can be approximated arbitrarily closely, with respect to \|\|$_{1}$, by functions from $C(S)$. Since $C(S)$ is a hyperplane in $C\left(S_{\infty}\right)$, with the constant functions on $S_{\infty}$ forming a one-dimensional supplementary manifold, what is required amounts to showing that every constant function can be so approximated and this we do now.

There exists a measurable set $P \in \mathbf{B}(S)$ on which the entire measure $\mu$ is concentrated, and an increasing sequence of compact sets $C_{1} \subset C_{2} \subset C_{3} \ldots$ whose union is $P$. Now let $\kappa$ be a constant function on $S_{\infty}$. Clearly we may assume without loss of generality that $\kappa>0$. Let $\kappa_{n}$ be a continuous function with compact support, which coincides with $\kappa$ on $C_{n}$ and has values between 0 and $\kappa$ elsewhere. Clearly $\left\|\kappa-\kappa_{n}\right\|_{1}$ tends to 0 . This completes the proof of our lemma. 
In much of what follows we shall need a criterion for the weak compactness of a set $K \subset C^{\prime}(S)$. Several such criteria were given by Grothendieck (11). However, the criterion most suitable for our purpose is one given by Bartle, Dunford, and Schwartz (1, Theorem 1.4, p. 292) for abstract measures on abstract $\sigma$-fields. On the other hand, it is not evident that if, everywhere in their theorem, one replaces the concept of abstract measure by our concept of topological measure and the concept of abstract $\sigma$-field by the concept of Borel class, the theorem remains valid. To show this is the purpose of the next theorem, which is a paraphrase, in the present setting, of the theorem of Bartle, Dunford, and Schwartz. We remind the reader that by a measure we mean here a regular Borel measure on $S$, and that we refer to other types of measures, in particular those used in (1), as abstract measures.

THeOREM 2.1. Let $K$ be a subset of $C^{\prime}(S)$. Then $K$ is relatively weakly compact (has weakly compact weak closure) if and only if it is bounded (i.e., the numbers $\bar{\mu}(S)$ remain inferior to a certain positive number $\eta$ as $\mu$ ranges over $K$ and there exists a non-negative measure $\lambda$ on $S$ such that $\mu(A)$ (equivalently $\bar{\mu}(A))$ tends to 0 , uniformly with respect to $\mu \in K$, as $\lambda(A)$ tends to 0 over the sets $A \in \mathbf{B}(S)$.

Proof. The fact that $\mu(A) \rightarrow 0$ is equivalent to $\bar{\mu}(A) \rightarrow 0$ follows from the inequality (8, p. 97, Lemma 5)

$$
\bar{\mu}(A) \leqslant 4 \sup |\mu(B)|,
$$

where the supremum is taken for all $B \in A$ and $B \in \mathbf{B}(S)$.

Let us first assume that $S$ is compact. Suppose that $K \in C^{\prime}(S)$ satisfies the condition of our theorem. Then $K$ satisfies the corresponding condition in (1, Theorem 1.4 , p. 297); hence it is weakly compact in the space of all finite abstract measures on $\mathbf{B}(S)$. It follows from this that $K$ is weakly compact in $C^{\prime}(S)$. Conversely, suppose that $K$ is weakly compact in $C^{\prime}(S)$. Then it is weakly compact in the space of all finite abstract measures on $\mathbf{B}(S)$. Therefore, we use again (1, Theorem 1.4, p. 292) to deduce the existence of an abstract measure $\lambda$ on $\mathbf{B}(S)$ with the desired property. Only this time we look at the construction of $\lambda$ in (1) and see that it is performed by means of a formula containing measures from $K$, which preserves their regularity. This means that $\lambda$ belongs to $C^{\prime}(S)$, as required by the present theorem.

Suppose that $S$ is locally compact, but not necessary compact. Now we utilize Lemma 2.1, and imbed $C^{\prime}(S)$ isometrically as a hyperplane of $C^{\prime}\left(S_{\infty}\right)$. Again we have that $K$ is weakly compact in $C^{\prime}(S)$ if and only if it is weakly compact in $C^{\prime}\left(S_{\infty}\right)$, if and only if there exists a $\lambda_{\infty} \in C^{\prime}\left(S_{\infty}\right)$ having the properties required by the theorem with respect to $K$ as a subset of $C^{\prime}\left(S_{\infty}\right)$. Now we have to examine the way in which $C^{\prime}(S)$ is a hyperplane of $C^{\prime}\left(S_{\infty}\right)$. Consider the point measure $\omega_{\infty}$ at $\infty$, with total mass $\lambda_{\infty}(\infty)$, and define $\lambda=\lambda_{\infty}-\omega_{\infty}$. Then $\lambda$ can be considered as a measure of $C^{\prime}(S)$, and it is easy to see that it has the desired properties. This completes the proof. 
3. Vector measures. Let $E$ stand, for a moment, for a general topological vector space, not necessarily a Banach space, and $m: \mathbf{B}(S) \rightarrow E$. We shall say that $m$ is an E-valued measure if $m$ is countably additive and regular. Countable additivity means that for every disjoint decomposition of any set $A \in \mathbf{B}(S)$ into sets $A_{1}, A_{2}, \ldots \in \mathbf{B}(S)$, we have

$$
m(A)=\sum_{i=1}^{\infty} m\left(A_{i}\right) .
$$

Obviously, the convergence of the series in (3.1) is, of necessity, unconditional. Regularity means (an obvious generalization to topological vector spaces of the definition (2) of Dinculeanu for Banach spaces) that, for every $A \in \mathbf{B}(S)$ and every neighbourhood $N$ of $0 \in E$, there exists an open measurable set $U$ and a compact set $C$ such that $S \supset U \supset A \supset C$, and $m(D) \in N$, for every measurable subset $D$ of $U \sim C$.

Let us return now to a Banach space $E$. We shall use the adjectives weak* (in case $E$ is a dual space), weak, and strong modifying the term $E$-measure to distinguish the various types of measures resulting from using on $E$ the various customary topologies. Our concept of strong $E$-valued measure is clearly the analogue for locally compact spaces $S$ of the concept of vector measure of Bartle, Dunford, and Schwartz (1), which they defined for abstract measurable spaces. It is easily seen that a mapping $m: \mathbf{B}(S) \rightarrow E^{\prime}$ is a weak* measure if and only if, for every $x \in E$, the scalar set function $\mu_{x}$, defined for $A \in \mathbf{B}(S)$ by

$$
\mu_{x}(A)=\langle x, m(A)\rangle,
$$

is a measure on $S$. Clearly a weak $E$-measure is a weak* $E^{\prime \prime}$-measure, all of whose values lie in $E$. For a weak measure $m: \mathbf{B}(S) \rightarrow E$ and $x^{\prime} \in E^{\prime}$, the measure $\mu_{x^{\prime}}$ is defined in a manner similar to (3.2), and belongs to $C^{\prime}(S)$. The next lemma is the topological analogue of (1, Lemma 2.2, p. 293). The only new feature is the fact, irrelevant in (1), that weak regularity implies strong regularity.

Lemma 3.1. A mapping $m: \mathbf{B}(S) \rightarrow E$ is a weak measure if and only if it is a strong measure.

Proof. The "if" part is trivial. Now we establish the "only if" part. Let $m$ be a weak measure. Strong countable additivity of $m$ follows from an argument that parallels that used in (1, Lemma 2.2, p. 293) almost word for word. We show that $m$ is strongly regular. In this connection we refer the reader to the definition of regularity at the beginning of this section. Consider the set $K$ of all measures $\mu_{x^{\prime}}$, described in the remarks preceding this theorem, for $\left\|x^{\prime}\right\| \leqslant 1$. The set $K \subset C^{\prime}(S)$ is relatively weakly compact. This can be established exactly as in (1, Lemma 2.3, p. 294). We conclude from Theorem 2.1 above that there exists a non-negative measure $\lambda$ on $S$ such that $\bar{\mu}_{x^{\prime}}(A)$ tends 
to 0 , uniformly in $\mu_{x^{\prime}} \in K$, as $\lambda(A)$ tends to 0 over the sets $A \in \mathbf{B}(S)$. Now let $\epsilon>0$. We know that there is a $\delta>0$ such that for $A \in \mathbf{B}(S)$ and $\lambda(A)<\delta$ we have $\bar{\mu}_{x^{\prime}}(A)<\epsilon$, for every $\mu_{x^{\prime}} \in K$. Since $\lambda$ is regular, for every $A \in \mathbf{B}(S)$, we can find a $U$ and a $C$, as in the definition of regularity, to go with $m=\lambda$ and the neighbourhood of 0 in the space of real numbers defined by $\delta$. We then have, for $D \in \mathbf{B}(S)$ such that $D \subset U \sim C$,

$$
\|m(D)\|=\sup \left|\mu_{x^{\prime}}(D)\right|
$$

where the supremum in (3.3) is taken for all $x^{\prime} \in E^{\prime},\left\|x^{\prime}\right\|=1$. This completes our proof.

In view of Lemma 2.1 the concepts of weak vector measure and strong measure coincide, and we use the term strong vector measure to refer to this common concept. Now let $m: \mathbf{B}(S) \rightarrow E^{\prime}$ be a weak* measure. As in (10), we define the semivariation of $m$, denoted by $\|m\|$, as

$$
\|m\|=\sup \bar{\mu}_{x}(S),
$$

where the supremum is taken as $x$ varies over the solid unit sphere of $E$. It follows easily from the closed-graph theorem that $\|m\|<+\infty$. In the special case of a strong measure $m: \mathbf{B}(S) \rightarrow E$, it is easy to verify that the semivariation reduces to the concept of semivariation introduced in (1) by an entirely different definition. We again use the notations $N\left(S, E^{\prime}\right)$ and $N_{s}(S, E)$ of (10) to denote, respectively, the space of weak* $E^{\prime}$-measures and the space of strong $E$-measures on $S$. These are Banach spaces under the semivariation norm.

Corollary 3.1. Every strong measure $m: \mathbf{B}(S) \rightarrow E$ can be extended to a strong measure $m_{\infty}: \mathbf{B}\left(S_{\infty}\right) \rightarrow E$ of the same semivariation, concentrated on $S$.

Proof. Let us consider the set $K$ of measures $\mu_{x^{\prime}}, x^{\prime} \in E^{\prime},\left\|x^{\prime}\right\| \leqslant 1$, introduced in the proof of Lemma 3.1 and the measure $\lambda$ obtained there. It is easy to see that $m$ is absolutely continuous with respect to $\lambda$. Now the extension is immediate, because as we have seen in $\$ 2$, there exists a measurable set $P$, on which $\lambda$, hence $m$, is concentrated. If we use the inclusion $P \subset S \subset S_{\infty}$, then for every measurable subset $B \subset S_{\infty}$, we let $m_{\infty}(B)=m(B \cap P)$. One verifies immediately that $m_{\infty}$ is indeed a strong $E$-measure that extends $m$ to the entire $S_{\infty}$. The preservation of the semivariation follows from the fact that the latter is defined in terms of the measures $\mu_{x^{\prime}}$, which themselves can be extended, preserving their total mass. This completes our proof.

It should be remarked that the result corresponding to the above corollary, for weak* measures, is obvious.

For any two Banach spaces $E$ and $F, \mathbf{L}(E, F)$ will always denote the Banach space of all bounded linear transformations of $E$ into $F$ with the transformation norm. We shall need below the following relation, discussed earlier in (10), 
but which we now simply state. The equation in it stands for an isometric isomorphism onto:

$$
N\left(S, E^{\prime}\right)=\mathbf{L}\left[C(S), F^{\prime}\right]
$$

If $m \in N\left(S, E^{\prime}\right)$ and $T_{m} \in \mathbf{L}\left[C(S), E^{\prime}\right]$ corresponds to $m$ under (3.5), then, for every $\phi \in C(S)$,

$$
T_{m} \phi=\int_{S} \phi(s) d m(s)
$$

where the integral can be defined in many equivalent ways, the simplest of which is by means of the following relation:

$$
\left\langle x, \int_{S} \phi(s) d m(s)\right\rangle=\int_{S} \phi(s) d \mu_{x}(s) .
$$

Obviously (3.7) defines the integral of (3.6) as an element of $E^{\prime}$. In the case of strong measures $m: B(S) \rightarrow E$, we do not have an equation corresponding to (3.5). However, we have the inclusion $N_{s}(S, E) \subset \mathbf{L}[C(S), E]$, which yields, corresponding to every $m \in N_{s}(S, E)$, a transformation $T_{m}: C(S) \rightarrow E$, given by (3.6). In this case, it is necessary to verify that the integral does indeed yield an element of $E$, but this is discussed well in (10) and we shall pursue the matter no further.

We discuss now a few examples of vector measures that ought to clarify matters somewhat. Strong vector measures have been in the literature for quite some time. Perhaps the simplest non-trivial examples of such measures are the unconditionally summable sequences of vectors $x_{1}, x_{2}, \ldots \in E$, i.e., the series $\sum_{i=1}^{\infty} x_{i}$ converges unconditionally. Here $S$ consists, of course, of the positive integers, and the vector measure is discrete. By the same token, a simple example of a weak* $E^{\prime}$-valued measure that is not a strong vector measure is obtained by using a sequence $x^{\prime}{ }_{1}, x^{\prime}{ }_{2}, \ldots$ of elements of $E^{\prime}$, which is weakly* unconditionally summable, but not strongly unconditionally summable. There are many examples of this. The simplest one is perhaps obtained in $\mathbf{1}_{\infty}=\left(\mathbf{1}_{1}\right)^{\prime}$ by taking $x^{\prime}{ }_{i}$ to be the sequence with 1 in the $i$ th place and 0 elsewhere. Later on we shall give a more interesting example by proving that the resolutions of the identity corresponding to certain spectral operators are weak* vector measures, which are not, in general, strong vector measures.

We now study the special case of strong $E$-valued measures on $S$ defined by Pettis' integrable mappings. Let $\rho$ be a non-negative measure on $S$, which for the present purpose need not be assumed to be finite, but is still assumed to be regular; and $f: S \rightarrow E$ a strongly $\rho$-measurable mapping. We shall say that $f$ is Pettis integrable $(\mathbf{1 5}$, p. $\mathbf{7 2})$ with respect to $\rho$ if, for every $x^{\prime} \in E^{\prime}$, the numerical function $\left\langle f(s), x^{\prime}\right\rangle$ of $s$ belongs to $L_{1}(\rho)$ and for every $A \in \mathbf{B}(S)$ there exists an element

$$
\int_{A} f(s) d \rho(s)
$$


of $E$ satisfying the condition

$$
\left\langle\int_{A} f(s) d(s), x^{\prime}\right\rangle=\int_{A}\left\langle f(s), x^{\prime}\right\rangle d \rho(s) .
$$

Clearly every Pettis integrable mapping $f$ defines a strong $E$-valued measure $m_{f}$ on $S$, via the formula

$$
m_{f}(A)=\int_{A} f(s) d \rho(s)
$$

for every $A \in \mathbf{B}(S)$. It therefore defines a bounded linear transformation $T_{f}: C(S) \rightarrow E$ by virtue of the formula (3.6) above. However, in this case, the transformation can be extended in a norm-preserving manner to a transformation that we continue to denote by $T_{f}$ of $L_{\infty}(\rho) \supset C(S)$ into $E$. For $\phi \in L_{\infty}(\rho)$ we have

$$
T_{f} \phi=\int_{S} \phi(s) f(s) d \rho(s) .
$$

The transformation $T_{f}: L_{\infty}(\rho) \rightarrow E$ was introduced by Pettis (18), who showed it to be compact. This was done for abstract measures $\rho$ defined on a $\sigma$-algebra of sets, but the results extend without difficulty to the present situation. It follows that $T_{f}: C(S) \rightarrow E$ is also compact. We can define a norm $\|f\|$ for a Pettis integrable mapping $f$ by means of the formula

$$
\|f\|=\sup \int_{S}\left|\left\langle f(s), x^{\prime}\right\rangle\right| d \rho(s)<+\infty
$$

where the supremum is taken for all unit functionals $x^{\prime}$ of $E^{\prime}$. This norm is also due to Pettis. We denote by $W(\rho, E)$ the completion of the space of all Pettis $\rho$-integrable mappings of $S$ into $E$, with respect to the norm (3.11). We state the next theorem without proof because its harder part is essentially due to Pettis (18).

THEOREM 3.1. There exist isometric isomorphisms, symbolized by the inclusions

$$
W(\rho, E) \subset N_{s}(S, E) \subset \mathbf{L}[C(S), E],
$$

in such a way that, if $f$ is a Pettis integrable mapping, and $m_{f} \in N_{s}(S, E)$ and $T_{f} \in \mathbf{L}[C(S), E]$ correspond to $f$ under these imbeddings, then $f$ and $m_{f}$ are related by formula (3.9) and f and $T_{f}$ by formula (3.10). If $m \in N_{s}(S, E)$ and $T_{m}$ corresponds to $m$ under the second imbedding in (3.12), then $m$ and $T_{m}$ are related by formula (3.6).

The part of Theorem 3.1 related to the second inclusion of (3.12) follows from an argument exactly analogous to that leading to the relation (3.50) above, except that here we obtain only inclusion instead of equality, and we shall soon see why. The part related to the first inclusion was established by 
Pettis for abstract measures, and his proof is easily adapted to the present situation. We observe also the following isometric inclusion:

$$
W(\rho, E) \subset \mathbf{L}\left[L_{\infty}(\rho), E\right] .
$$

That the first inclusion in Theorem 3.1 is proper, in general, follows easily from the fact that $W(\rho, E)$ contains only measures that are absolutely continuous with respect to $\rho$, while $N_{s}(S, E)$ may contain others, as can be easily seen, even in the case when $E$ is the field of scalars itself. To show that the second inclusion in (3.12) can be proper, one can utilize the equation (3.5), which yields that, when $E$ is replaced by $E^{\prime}$ in (3.12), we obtain

$$
N_{s}\left(S, E^{\prime}\right) \subset N\left(S, E^{\prime}\right)=\mathbf{L}\left[C(S), E^{\prime}\right]
$$

from which it follows that we shall have a proper inclusion every time we can exhibit a weak* $E^{\prime}$-measure that is not a strong measure. This has already been done in this section.

It follows from Theorem 3.1 that the individual elements of $W(\rho, S)$, limits under the natural norm, of sequences of Pettis integrable mappings, can be considered as measures. It would be more satisfying if we had a theorem that stated that the limit of Pettis integrable mappings is a Pettis integrable mapping. However, so far as we know, the possibility of the existence of such a theorem is an open question. In its absence a criterion to tell when a Cauchy sequence in $W(\rho, E)$ of Pettis $\rho$-integrable mappings converges to a Pettis $\rho$-integrable mapping may be of interest. Such a criterion is contained in the next theorem, which is a sort of bounded-convergence theorem for Pettis integrable mappings. We can only establish the result under rather restrictive conditions. Accordingly we assume that $E$ is reflexive and separable. It follows that $E^{\prime}$ is separable as well, and contains a dense countable subset $x^{\prime}{ }_{1}, x^{\prime}{ }_{2}, \ldots$ Now let $f_{1}, f_{2}, \ldots$ be a sequence of Pettis $\rho$-integrable mappings of $S$ into $E$. For each positive integer $j$, let

$$
S_{j}=\left\{s \mid s \in S,\left\|f_{n}(s)\right\|<j \text { for every } n\right\} .
$$

THEOREM 3.2. Let E be a reflexive separable Banach space and $\rho$ a non-negative measure (which need not be finite, but is still assumed to be regular) on S. Suppose $f_{1}, f_{2}, \ldots$ is a sequence of Pettis $\rho$-integrable mappings from $S$ into $E$, which is a Cauchy sequence in the space $W(\rho, E)$. A ssume further that the complement of the set $\cup_{j=1}^{\infty} S_{j}$ is a null set. Then the sequence $f_{1}, f_{2}, \ldots$ converges in $W(\rho, E)$ to a Pettis $\rho$-integrable mapping $f: S \rightarrow E$.

Proof. By passing to a subsequence, we can assume that $\left\|f_{n+1}-f_{n}\right\|<1 / 2^{n}$ and, by a familiar argument, that, for every $s$ outside of a set of measure zero and for every $k$, the sequence $\left\langle f_{1}(s), x^{\prime}{ }_{k}\right\rangle,\left\langle f_{2}(s), x^{\prime}{ }_{k}\right\rangle, \ldots$ of numerically valued functions converges to a function $\phi_{k}(s) \in L_{1}(\rho)$. Let us denote by $\widetilde{S}$ the 
complement of the null set in question. Without loss of generality, we may assume that

$$
\widetilde{S}=\bigcup_{j=1}^{\infty} S_{j},
$$

since both these sets have null complements. We are going to show that for every $s \in \widetilde{S}$ and every $x^{\prime} \in E^{\prime}$, the sequence $\left\langle f_{1}(s), x^{\prime}\right\rangle,\left\langle f_{2}(s), x^{\prime}\right\rangle, \ldots$ converges to a function belonging to $L_{1}(\rho)$. Let us fix $x^{\prime} \in E^{\prime}$ and $s \in \widetilde{S}$. Then $s$ belongs to some $S_{j}$, which we fix for the purpose of the following calculation. We have, for arbitrary $f_{n}$ and $f_{m}$,

$$
\begin{aligned}
& \left|\left\langle f_{m}(s), x^{\prime}\right\rangle-\left\langle f_{n}(s), x^{\prime}\right\rangle\right| \\
& \quad \leqslant\left|\left\langle f_{m}(s), x^{\prime}-x^{\prime}{ }_{k}\right\rangle\right|+\left|\left\langle f_{n}(s), x^{\prime}-x^{\prime}{ }_{k}\right\rangle\right|+\left|\left\langle f_{m}(s)-f_{n}(s), x^{\prime}{ }_{k}\right\rangle\right| .
\end{aligned}
$$

Now, from the fact that $s \in S_{j}$, we have

$$
\left|\left\langle f_{m}(s), x^{\prime}\right\rangle-\left\langle f_{n}(s), x^{\prime}\right\rangle\right| \leqslant 2|| x^{\prime}-x^{\prime}{ }_{k} \| j+\left|\left\langle f_{m}(s)-f_{n}(s), x^{\prime}{ }_{k}\right\rangle\right| .
$$

Now the first term on the right of (3.15) can be made arbitrarily small for suitable $k$. On the other hand, for that $k$ the last term can be made arbitrarily small for $m$ and $n$ sufficiently large, because each sequence $\left\langle f_{1}(s), x^{\prime}{ }_{k}\right\rangle$, $\left\langle f_{2}(s), x^{\prime}{ }_{k}\right\rangle, \ldots$ converges to $\phi_{k}(s)$. From this it follows that, for every $s \in \widetilde{S}$ and every $x^{\prime}$ in $E^{\prime}$, the sequence $\left\langle f_{1}(s), x^{\prime}\right\rangle,\left\langle f_{2}(s), x^{\prime}\right\rangle, \ldots$ converges to a number which we denote by $\left\langle f(s), x^{\prime}\right\rangle$. For each $s \in \widetilde{S},\left\langle f(s), x^{\prime}\right\rangle$ is linear in $x^{\prime}$, being the weak limit of linear functionals. One now shows by means of manipulations similar to those employed in (3.14) and (3.15) that for every $s \in S_{j}$, $\left|\left\langle f(s), x^{\prime}\right\rangle\right| \leqslant j|| x^{\prime} \|$, for every $x^{\prime} \in E^{\prime}$; hence $f(s) \in E$, since $E$ is reflexive. Now, from the Cauchy condition on the sequence $f_{1}, f_{2}, \ldots$ in the sense of $W(\rho, E)$, it follows that each sequence $\left\langle f_{1}(s), x^{\prime}\right\rangle,\left\langle f_{2}(s), x^{\prime}\right\rangle, \ldots$ is a Cauchy sequence of $L_{1}(\rho)$; hence $\left\langle f(s), x^{\prime}\right\rangle$ as a function of $s$ (defined arbitrarily outside of the set $\widetilde{S}$ ) represents an element of $L_{1}(\rho)$. We have thus obtained the values of the mapping $f: S \rightarrow E$ for $s \in \widetilde{S}$ and can define it arbitrarily on the null complement of $\widetilde{S}$.

We now show that $f$ is Pettis $\rho$-integrable. We fix $A \in \mathbf{B}(S)$. For $x^{\prime} \in E^{\prime}$,

$$
\int_{A}\left|\left\langle f(s), x^{\prime}\right\rangle\right| d \rho(s) \leqslant \int_{S}\left|\left\langle f(s), x^{\prime}\right\rangle\right| d \rho(s)
$$

the existence of the latter integral having been established above. Furthermore, both integrals are dominated by $\left\|x^{\prime}\right\| \sup n\left\|f_{n}\right\|$. (We remind the reader that $\left\|f_{n}\right\|$ is defined as the norm of the element $f_{n} \in W(\rho, E)$, by means of (3.11).) Hence, the integral

$$
\int_{A}\left\langle f(s), x^{\prime}\right\rangle d \rho(s)
$$

defines a bounded linear functional in $x^{\prime}$, hence an element of $E$. This shows that $f$ is Pettis integrable. 
We now show that $\left\|f-f_{n}\right\|$ tends to zero as $n$ tends to $\infty$. The mapping $f$ is considered an element of $W(\rho, E)$, when one identifies it with the strong measure $m_{f}$ which it defines. Now the Cauchy sequence $f_{1}, f_{2}, \ldots$ converges to a measure $m$ of $W(\rho, E)$. Hence our assertion follows from a verification that $m$ coincides with $m_{f}$. But this can be done by means of a direct straightforward calculation. This completes our proof.

As we have indicated earlier, the condition that the complement of the union $\cup_{j=1}^{\infty} S_{j}$ be null is a sort of bounded convergence condition, and is satisfied, for instance, when there exists a function $\phi \in L_{1}(\rho)$ such that $\left\|f_{k}(s)\right\| \leqslant \phi(s)$ for almost every $s$ and every $k$.

4. Vector measures of finite variation. Let us now consider a weak* vector measure $m: \mathbf{B}(S) \rightarrow E^{\prime}$. The main purpose of this section is to prove a theorem similar to the Pettis theorem (Lemma 3.1) discussed in $\$ 3$. It states that although the class of strong $E^{\prime}$-valued measures is a proper subclass of the class of all weak ${ }^{*} E^{\prime}$-valued measures, we have that a weak* $E^{\prime}$-valued measure is a strong vector measure, provided that its total variation is finite. In (10) we have used the following definition of the variation $\mu^{m}$ of $m$ which was given by Dinculeanu in (2). For $A \in \mathbf{B}(S), \mu^{m}(A)$ is given by

$$
\mu^{m}(A)=\sup \sum_{i=1}^{n}\left\|m\left(A_{i}\right)\right\|
$$

where the supremum is taken over all the decompositions of $A$ into disjoint sets $A_{1}, A_{2}, \ldots, A_{n}, A_{i} \in \mathbf{B}(S)$. It was shown by Dinculeanu (3) that $\mu^{m}$ is an abstract measure (i.e., countably additive) at least when $S$ is compact, $m$ is a strong measure, and $\mu^{m}(S)<+\infty$. However, his proof extends easily to the completely general case considered here. In the present situation, of course, one can only conclude that $\mu^{m}$ is an abstract, not necessarily finite, positive measure. The question of regularity of $\mu^{m}$ will be discussed shortly. We shall say that $m$ has finite variation if $\mu^{m}$ is a finite abstract measure.

TheOREM 4.1. Let $m: \mathbf{B}(S) \rightarrow E^{\prime}$ be a weak* measure on a locally compact Hausdorff space $S$, such that $m$ has finite variation $\mu^{m}$. Then $m$ is a strong measure and $\mu^{m}$ is a non-negative measure (i.e. finite and regular).

Proof. Let $A_{1}, A_{2}, \ldots$ be a sequence of disjoint sets from $\mathbf{B}(S)$, and

We show that

$$
A=\bigcup_{i=1}^{\infty} A_{i}
$$

$$
m(A)=\sum_{i=1}^{\infty} m\left(A_{i}\right)
$$

In the first place we know that $m$ is finitely additive. This follows directly from its being a weak* measure and has nothing to do with its having finite 
variation. We also know that $\|m(B)\| \leqslant \mu^{m}(B)$, for every $B \in \mathbf{B}(S)$. Now let $\epsilon>0$. There is a positive integer $N$ such that, for $n>N, \mu^{m}\left(A \sim \cup_{i=1}^{n} A_{i}\right)<\epsilon$. We now have, using the finite additivity of $m$,

$$
\begin{aligned}
\| m(A) & -\sum_{i=1}^{n} m\left(A_{i}\right)\|=\| m\left(A \sim \bigcup_{i=1}^{n} A_{i}\right) \| \\
& \leqslant \mu^{m}\left(A \sim \bigcup_{i=1}^{n} A_{i}\right)<\epsilon .
\end{aligned}
$$

This concludes the proof of the strong countable additivity of $m$.

We now show that $m$ is strongly regular. Let $K$ be the set of all measures $\mu_{x}$ (see $\S 3$ ) for $x$ in the solid unit sphere of $E$. Now every measure $\mu_{x} \in K$ can be extended to a measure on the one-point compactification $S_{\infty}$ of $S$, concentrated on $S$. The new measures form a set $K_{\infty}$. The finite abstract non-negative Borel measure $\mu^{m}$ on $S$ can also be extended to a finite abstract non-negative Borel measure $\mu_{\infty}{ }^{m}$ on $S_{\infty}$ concentrated on $S$, in the manner indicated in Lemma 2.2. One sees easily that $\mu_{\infty}{ }^{m}$ satisfies the conditions required of $\nu$ in (1, Theorem 1.4, p. 292). From this we conclude that $K_{\infty}$ is a relatively weakly compact subset of $C^{\prime}\left(S_{\infty}\right)$; hence $K$ is a relatively weakly compact subset of $C^{\prime}(S)$. By Theorem 2.1 , there exists a non-negative measure $\lambda$ on $S$ (that is, $\lambda$ is a finite regular Borel measure on $S$ ) such that $\bar{\mu}_{x}(A)$ tends to 0 uniformly with respect to $\mu_{x} \in K$, as $\lambda(A)$ tends to 0 over the sets of $\mathbf{B}(S)$. From this point on, the proof of strong regularity of $m$ follows the pattern of a similar argument in Lemma 3.1 and the details are omitted.

We see from Theorem 4.1 that, for mappings $m: \mathbf{B}(S) \rightarrow E^{\prime}$ of finite variation, the distinction between weak* and strong vector measure disappears and that the fact that the values $m(A)$ lie in $E^{\prime}$, a dual space, is irrelevant. Consequently we shall refer to these measures simply as vector measures of finite variation and, when convenient, shall use them in the form $m: \mathbf{B}(S) \rightarrow E$ for a perfectly general Banach space $E$. As in (10), we denote by $M(S, E)$ the space of all vector measures $m: \mathbf{B}(S) \rightarrow E$ of finite variation, which can easily be shown to be a Banach space under the norm given by the total variation $\mu^{m}(S)$.

Theorem 4.1 can be used to fill gaps in the proof of a theorem of Ivan Singer (20), stating the existence of an isometric isomorphism represented by the equation

$$
C^{\prime}(S, E)=M\left(S, E^{\prime}\right)
$$

a generalization of the Riesz representation theorem (17). In (4.4), $C^{\prime}(S, E)$ stands for the dual space of the space $C(S, E)$ of all continuous mappings $g: S \rightarrow E, S$ compact and Hausdorff. One gap in the proof occurs in the statement (see the last paragraph of p. 303 in 20) that weak* (which he calls weak) countable additivity implies strong countable additivity. We have seen in $\$ 3$ that this statement is not quite correct; and in (20) it is the result of an incorrect application of the Pettis theorem discussed above in $\S 3$. The statement 
is used in the proof of (4.4) to conclude that a weak* measure $m_{g^{\prime}}: \mathbf{B}\left(S^{\prime}\right) \rightarrow E^{\prime}$, constructed out of a bounded linear functional $g^{\prime} \in C^{\prime}(S, E)$, is actually strongly countably additive. Fortunately, it has been established that $m_{g^{\prime}}$ has finite variation, and strong countable additivity follows from Theorem 4.1 above. Again, although the regularity actually established for $m_{g^{\prime}}$ in (20) is only in the weak* sense (see the definition at the bottom of p. 305 in 20 ), we may conclude strong regularity from our Theorem 4.1 .

5. Vector measures defined by Bochner integrable mappings. In this section we present a preliminary discussion of one of the central questions that will occupy our attention in $\S 6$ of the present work. The question is the following. We know that a measure $m: \mathbf{B}(S) \rightarrow E$ of finite variation is absolutely continuous with respect to its variation measure $\mu^{m}$. This means that, for every $A \in \mathbf{B}(S), \mu^{m}(A)=0$ implies that $m(A)=0$. One then wonders whether $m$ might have, in some sense, a Radon-Nikodym derivative with respect to $\mu^{m}$, i.e., a mapping $h_{m}: S \rightarrow E$ for which the necessary integrals exist and satisfy the relation

$$
m(A)=\int_{A} h_{m}(s) d \mu^{m}(s)
$$

for every $A \in \mathbf{B}(S)$. Whatever information is available on this question will be discussed in $\$ 6$. In particular we shall see by means of an example that if one insists that $h_{m}$ be Bochner integrable with respect to $\mu^{m}$, the answer is, in general, negative. In this section we limit ourselves to the discussion of two elementary cases where the answer is affirmative. These, in turn, will help in the discussion of the more delicate questions considered in $\$ 6$.

Now let $\rho$ be any non-negative (not necessarily finite) measure on $S$. We shall employ the term Bochner integrable, to describe a mapping $f: S \rightarrow E$, in the sense used by Hille and Phillips (15, p. 79). Since a Bochner $\rho$-integrable mapping $f: S \rightarrow E$ is Pettis $\rho$-integrable, it defines, according to our discussion in $\S 3$, a vector measure $m_{f}: \mathbf{B}(S) \rightarrow E$ given, for $A \in \mathbf{B}(S)$, by

$$
m_{f}(A)=\int_{A} f(s) d \rho(s) .
$$

TheOREM 5.1. Let $\rho$ be a non-negative (not necessarily finite) measure on $S$, and $f: S \rightarrow E$ a Bochner $\rho$-integrable mapping. Then the vector measure $m_{f}$ has finite variation, $\mu^{m_{f}}$ is given, for $A \in \mathbf{B}(S)$, by

$$
\mu^{m_{f}}(A)=\int_{A}\|f(s)\| d \rho(s),
$$

and $m_{f}$ has representation (5.1) in terms of $\mu^{m_{f}}$, where $h_{m}(s)=f(s) /\|f(s)\|$, for every $s$ for which $f(s) \neq 0$ and $h_{m}(s)=0$ for $f(s)=0$. Furthermore, $h_{m}$ is Bochner integrable with respect to $\mu^{m_{f}}$. 
Proof. It follows easily from (5.2) that

$$
\mu^{m_{f}}(A) \leqslant \int_{A}\|f(s)\| d \rho(s),
$$

for every $A \in \mathbf{B}(S)$. From this and the Bochner integrability of $f$, it follows that $m_{f}$ has finite variation.

We now establish formula (5.3). Let us, as usual, denote by $L_{1}(\rho, E)$ the Banach space of all Bochner $\rho$-integrable mappings $f: S \rightarrow E$, with the norm

$$
\|f\|_{1}=\int_{S}\|f(s)\| d \mu(s) .
$$

Now it is easy to establish (5.3) if $f$ is a simple (i.e., taking on a finite number of values) Bochner-integrable mapping. We know also from the theory of the Bochner integral that such mappings are dense in $L_{1}(\rho, E)$. Now let $f$ be an arbitrary element of $L_{1}(\rho, E) ; f_{1}, f_{2}, \ldots$ a sequence of simple mappings converging to $f$ in $L_{1}(\rho, E)$; and $m_{1}, m_{2}, \ldots$ the corresponding sequence of vector measures defined by the $f_{i}$ 's according to (5.2). Then, for each $m_{i}$ and $f_{i},(5.3)$ is verified. Let $\epsilon$ be an arbitrary positive number. Then, for some $i$,

$$
\left\|f-f_{i}\right\|_{1}<\epsilon .
$$

It follows from (5.4) that also

$$
\left|\mu^{m_{f}}(A)-\mu^{m_{i}}(A)\right|<\epsilon .
$$

We then have

$$
\begin{aligned}
\int_{A}\|f(s)\| d \rho(s) & \leqslant \int_{A}\left\|f_{i}(s)\right\| d \rho(s)+\epsilon \\
& \leqslant \mu^{m_{i}}(A)+\epsilon=\mu^{m_{f}}(A)+2 \epsilon .
\end{aligned}
$$

Now (5.3) follows from (5.4) and (5.5).

We now verify the required expression for $h_{m}$ given in our theorem. We denote by $f(s) /\|f(s)\|$ the mapping given by that ratio when the ratio makes sense, and given by zero otherwise. Since that mapping is bounded, to show that it is

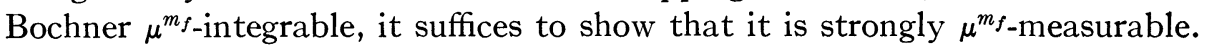
We do this. In the first place, since $f$ is strongly $\rho$-measurable and $\mu^{m_{f}}$ is absolutely continuous with respect to $\rho$, it follows that $f$ is strongly $\mu^{m_{f} \text {-measurable. }}$ Now, if $E$ is the field of scalars, the measurability of $f(s) /\|f(s)\|$ with respect to $\mu^{m_{f}}$ is a classical result. It follows then that, for general $E, f(s) /\|f(s)\|$ is weakly $\mu^{m_{f}}$-measurable. We now use a theorem of Pettis $(\mathbf{1 8}$, p. 278 , Theorem

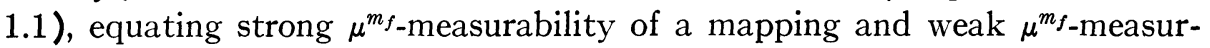

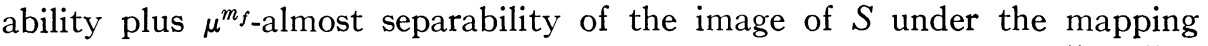
(see the discussion in 15). We have that the image of $S$ under $f(s) /\|f(s)\|$ is

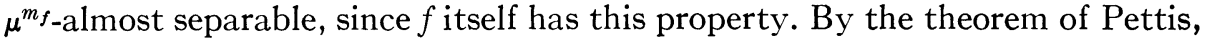




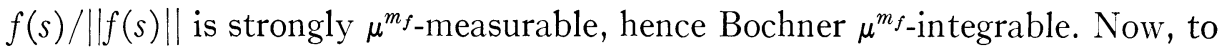
establish (5.1), all we need is to establish (see (5.3)) the equation

$$
\int_{A} f(s) d \rho(s)=\int_{A} f(s) /\|f(s)\| d \mu^{m_{f}}(s),
$$

for every $A \in \mathbf{B}(S)$. But this vector equation can be reduced to an equation involving ordinary scalar integrals by letting both sides be acted upon by an arbitrary $x^{\prime} \in E^{\prime}$. Then the equality follows from the ordinary Radon-Nikodym theorem. This completes the proof of our theorem.

Theorem 5.1 is a classical theorem in the case of scalar-valued mappings, but we have not seen the theorem in the literature for vector-valued mappings.

We now consider another situation in which the representation (5.1) is available for vector measures of finite variation. In (10) we have defined a Banach space $E$ to be a Phillips space if for any compact Hausdorff space $S$, any non-negative measure $\mu$ on $S$, and any bounded linear transformation $T: L_{1}(\mu) \rightarrow E$, there exists a Bochner $\mu$-integrable mapping $h: S \rightarrow E$, such that

$$
\|T\|=\underset{s \in S}{\operatorname{ess} \sup }\|h(s)\|
$$

and, for every $\phi \in L_{1}(\mu)$,

$$
T \phi=\int_{S} \phi(s) h(s) d \mu(s) .
$$

This definition is a modification of one due to Grothendieck (12) whose relation to the present one is explained in (10). Reflexive spaces, as well as separable duals of Banach spaces, are Phillips spaces. We shall now show that in the definition of Phillips spaces we could have dispensed with the requirement that

$$
{ }_{1} T\|=\underset{s \in S}{\operatorname{ess} \sup }\| h(s) \| .
$$

Lemma 5.1. Let $S$ be any locally compact Hausdorf space, $\mu$ a non-negative measure on $S$, and $h: S \rightarrow E$ a strongly $\mu$-measurable mapping. Suppose that, for every $\phi \in L_{1}(\mu)$, the mapping given at $s$ by $\phi(s) h(s)$ is Pettis $\mu$-integrable and that the linear transformation $T: L_{1}(\mu) \rightarrow E$, defined by the relation

$$
T \phi=\int_{S} \phi(s) h(s) d \mu(s),
$$

is bounded. Then $h$ is essentially $\mu$-bounded and

$$
\|T\|=\underset{s \in S}{\operatorname{ess} \sup }\|h(s)\| .
$$

Proof. The fact that

$$
\|T\| \leqslant \operatorname{ess} \sup _{s \in S}\|h(s)\|
$$


follows directly from (5.8) and details are omitted. What remains to be proved is the reverse inequality. We know that, since $h$ is strongly $\mu$-measurable, there exists a subset $S_{1} \subset S$, with null complement such that the image $h\left(S_{1}\right) \subset E$ is separable. Consequently, we may as well assume that $h(S)$ is separable or, equivalently, that $E$ is separable. It follows that the solid unit sphere of $E^{\prime}$ is weakly* separable; hence it contains a countable weakly* dense subset $x^{\prime}{ }_{1}, x_{2}^{\prime}, \ldots$ Now let us show that

$$
\text { ess sup }\|h(s)\| \leqslant\|T\| \text {. }
$$

It suffices to show that for every $\kappa>\|T\|,\|h(s)\| \leqslant \kappa$ a.e. with respect to $\mu$. Let $\kappa>\|T\|$. For fixed $x^{\prime}{ }_{k}$, the expression $\left\langle T \phi, x_{k}^{\prime}{ }_{k}\right\rangle$, for $\phi \in L_{1}(\mu)$, defines a bounded linear functional on $L_{1}(\mu)$. This functional, according to the duality theory of $L_{1}(\mu)$, is given by a function of $L_{\infty}(\mu)$, which in this case coincides with $\left\langle h(s), x^{\prime}{ }_{k}\right\rangle$ a.e. It then follows easily that, almost everywhere with respect to $\mu,\left|\left\langle h(s), x^{\prime}{ }_{k}\right\rangle\right| \leqslant\|T\|$ since $\|T\|$ dominates the norm of each functional $\left\langle T \phi, x^{\prime}{ }_{k}\right\rangle$. We then have, for every $x^{\prime}{ }_{k}$ and almost every $s$,

$$
\left|\left\langle h(s), x^{\prime}{ }_{k}\right\rangle\right| \leqslant\|T\|<\kappa ;
$$

hence $\|h(s)\|<\kappa$, a.e. with respect to $\mu$. This completes our proof.

Since in this work we are dealing with locally compact spaces $S$ that may not be compact, we must extend to such spaces the representation (5.7), given only for compact spaces.

Theorem 5.2. Let $E$ be a Phillips space, $S$ a locally compact Hausdorff space, $\mu$ a finite non-negative measure on $S$, and $T: L_{1}(\mu) \rightarrow E$ a bounded linear transformation. Then there exists a strongly $\mu$-measurable mapping $h: S \rightarrow E$, satisfying (5.7) and such that

$$
\|T\|=\underset{s \in S}{\operatorname{ess} \sup }\|h(s)\| .
$$

Proof. In this proof we utilize the material developed in $\$ 2$ concerning the extension of measures from $S$ to its one-point compactification $S_{\infty}$. Let us consider the extension $\mu_{\infty}$ of $\mu$ to $S_{\infty}$. We have now from Lemma 2.2 that $L_{1}(\mu)$ can be identified isometrically with $L_{1}\left(\mu_{\infty}\right)$ in the natural way explained in the proof of that lemma. We shall use the properties of this identification in the present proof. $T$ can then be considered as a bounded linear transformation of $L_{1}\left(\mu_{\infty}\right)$ into $E$. Since $E$ is a Phillips space and $S_{\infty}$ is compact, there exists a mapping $h_{\infty}: S_{\infty} \rightarrow E$ having the properties demanded of $h$ in the theorem, only in the context of $S_{\infty}$. We know that there exists a measurable subset $P$ of $S$ on which the entire measure $\mu$ is concentrated. We now define $h=\chi_{P} h_{\infty}$, where $\chi_{P}$ is the characteristic function of $P$. We consider $h$ as a mapping of $S$ into $E$. This mapping $h$ is the one required by the theorem. The fact that $h$ is strongly $\mu$-measurable is somewhat delicate, and we shall discuss it in a moment. The other properties required of $h$ by the theorem are easy to verify 
and we omit the details. Let us look at the strong measurability. In discussing it, we use again the theorem of Pettis (18) quoted in the proof of Theorem 5.1 above equating strong measurability with the combination of weak measurability and the almost separability of the image. The fact that $h_{\infty}(S)$ is $\mu_{\infty}$ almost separable implies immediately the corresponding property for $h(S)$ with respect to $\mu$. The weak measurability of $h$ with respect to $\mu$ follows from that of $h_{\infty}$ with respect to $\mu_{\infty}$ and the fact that the intersection of a $\mu_{\infty}$-measurable subset of $S_{\infty}$ with $S$ is $\mu$-measurable. This completes our proof.

Theorem 5.3. Let $E$ be a Phillips space and $m: \mathbf{B}(S) \rightarrow E$ a vector measure of finite variation. Then $m$ has a representation (5.1), where $h_{m}$ is strongly Bochner $\mu^{m}$-integrable.

Proof. We define the linear transformation $T: L_{1}\left(\mu^{m}\right) \rightarrow E$ by means of the equation

$$
T \phi=\int_{S} \phi(s) d m
$$

for $\phi \in L_{1}\left(\mu^{m}\right)$. The integral on the right of (5.9) has a meaning, as proved in (5) and discussed in (10). It also follows that $T$ is bounded by $\mu^{m}(S)$. It follows from Theorem 5.2 that $T$ has an integral representation

$$
T \phi=\int_{S} \phi(s) h_{m}(s) d \mu^{m}(s)
$$

for $\phi \in L_{1}\left(\mu^{m}\right)$, where $h_{m}: S \rightarrow E$ is Bochner $\mu^{m}$-integrable. Now (5.1) follows from (5.10), if we let $\phi$ in (5.10) be the characteristic function of $A$. This completes our proof.

6. Measures and tensors. In this section we discuss the relation between tensor products and vector measures, in certain cases clarifying the nature of the vector measures discussed above, in others obtaining representations of tensor products as spaces of measures. In particular, we find the tensor notation advantageous in discussing theorems of the Radon-Nikodym type. We begin our discussion with a very brief presentation of the theory of topological tensor products, as it applies to the problems that interest us here. A more extensive discussion can be found in (10), as well as in the works of Schatten (19) and Grothendieck (12).

Let $E$ and $F$ be, as before, Banach spaces. A tensor $\mathrm{t} \in F \otimes E$ has the form

$$
\mathrm{t}=\sum_{i=1}^{n} y_{i} \otimes x_{i}
$$

where $y_{i} \in F$ and $x_{i} \in E$.

The greatest cross norm $\gamma(t)$ of a tensor t having at least one representation of the form (6.1) is given by

$$
\gamma(t)=\inf \sum_{i=1}^{n}\left\|y_{i}\right\|\left\|x_{i}\right\|
$$


where the infimum is taken over all possible representations (6.1) of t. The completion of $F \otimes E$ with respect to the norm $\gamma$ is denoted by $F \otimes_{\gamma} E$. By a theorem of Grothendieck (12, Theorem 1, p. 51), every $\mathrm{t} \in F \otimes_{\gamma} E$ has a representation

$$
\mathrm{t}=\sum_{i=1}^{\infty} y_{i} \otimes x_{i}
$$

where the series converges absolutely in $F \otimes_{\gamma} E$ and

$$
\gamma(t)=\inf \sum_{i=1}^{\infty}\left\|y_{i}\right\|\left\|x_{i}\right\|<+\infty .
$$

In (6.4) the infimum is taken over all possible representations of $t$ of the form (6.3). By a theorem of Schatten (19, Theorem 3.2, p. 47), there exists an isometric isomorphism represented by the equation

$$
\left(F \otimes_{\gamma} E\right)^{\prime}=\mathbf{L}\left(F, E^{\prime}\right)
$$

where the space on the left is the dual space of $F \otimes_{\gamma} E$ and $\mathbf{L}\left(F, E^{\prime}\right)$ (see $\S 3)$ the space of all bounded linear transformations $T: F \rightarrow E^{\prime}$.

A tensor $\mathrm{t}$ of the form (6.3) defines, in a natural way, a bounded linear transformation $T^{t}: F^{\prime} \rightarrow E$, given, for $y^{\prime} \in F^{\prime}$, by

$$
T^{\mathrm{t}} y^{\prime}=\sum_{i=1}^{\infty}\left\langle y_{i}, y^{\prime}\right\rangle x_{i}
$$

In particular $T^{\mathfrak{t}}$ is well defined for the finite tensors of the algebraic tensor product $F \otimes E$. For such a tensor, the least cross norm $\lambda(t)$ is defined as $\lambda(t)=\left\|T^{t}\right\|$. The completion of $F \otimes E$ with respect to $\lambda$ is denoted by $F \otimes_{\lambda} E$ and can be identified isometrically with the subspace of $\mathbf{L}\left(F^{\prime}, E\right)$ consisting of all continuous linear transformations of $F^{\prime}$ (with the weak* topology) into $E$, that can be approximated uniformly by bounded linear transformations of finite rank. Under this identification, to every $\mathfrak{t} \in E \otimes_{\lambda} E$ corresponds a $T^{\mathfrak{t}} \in \mathbf{L}\left(F^{\prime}, E\right)$. Clearly the mapping $\mathrm{t} \rightarrow T^{\mathfrak{t}}$ thus defined is the extension to the entire $F \otimes_{\lambda} E$ of the mapping $t \rightarrow T^{\mathrm{t}}$ defined above on $F \otimes E$.

In this section we shall consider the two tensor products $C^{\prime}(S) \otimes_{\gamma} E$ and $L_{1}(\rho) \otimes_{\lambda} E$ for a not necessarily finite (but still regular) measure $\rho$ on $S$. We have already shown in (10) that the first tensor product is a space of vector measures of finite variation. Here we present a more detailed study of its structure. But first we shall discuss the second of these two tensor products and show that it is a space of strong vector measures, namely $W(\rho, E)$.

Applying the above general discussion of tensor products to $L_{1}(\rho) \otimes_{\lambda} E$, we obtain, for every $\mathrm{t} \in L_{1}(\rho) \otimes_{\lambda} E$ a bounded linear transformation $T^{\mathfrak{t}}: L_{\infty}(\rho) \rightarrow E$. For the special case of a tensor $\mathrm{t} \in L_{1}(\rho) \otimes_{\lambda} E$ with a representation

$$
\mathrm{t}=\sum_{i=1}^{\infty} \phi_{i} \otimes x_{i}
$$


$\phi_{i} \in L_{1}(\rho), x_{i} \in E$, satisfying (6.4), we can write explicitly, for $\psi \in L_{\infty}(\rho)$,

$$
T^{\mathrm{t}} \psi=\sum_{i=1}^{\infty} x_{i} \int_{S} \phi_{i}(s) \psi(s) d \rho(s) .
$$

We can also define explicitly, for such a tensor, a mapping $f^{t}: S \rightarrow E$, given, for $s \in S$, by

$$
f^{\mathrm{t}}(s)=\sum_{i=1}^{\infty} \phi_{i}(s) x_{i}
$$

This mapping is easily seen to be Bochner integrable, hence Pettis integrable, with respect to $\rho$. It follows from our discussion in $\$ 5$ (see, in particular, Theorem 5.1) that $f^{\mathfrak{t}}$ determines a vector measure $m^{\mathfrak{t}}: \mathbf{B}(S) \rightarrow E$ of finite variation. In particular, $m^{t}$ is a strong vector measure. These considerations are valid, in particular, in the special case in which the tensor $t$ of (6.7) reduces to a finite tensor (i.e., an element of the algebraic tensor product $L_{1}(\rho) \otimes E$ ). For such tensors one verifies readily that any one of the objects $t, T^{\mathrm{t}}, f^{\mathrm{t}}, m^{\mathrm{t}}$ determines the others uniquely.

Theorem 6.1. Let $\rho$ be a positive not necessarily finite measure on $S$. Then there exists an isometric isomorphism, represented by the equation

$$
L_{1}(\rho) \otimes_{\lambda} E=W(\rho, E)
$$

such that, if $m^{\mathrm{t}} \in W(\rho, E)$ corresponds to $\mathrm{t} \in L_{1}(\rho) \otimes E$ under the isomorphism, we have, for every $\psi \in L_{\infty}(\rho)$,

$$
T^{\mathrm{t}} \psi=\int_{S} \psi(s) d m^{\mathrm{t}}(s) .
$$

The restriction of $T^{\mathrm{t}}$ to $C(S)$ coincides with the transformation $T_{m}$ obtained in (3.6) upon replacing $m$ there by $m^{t}$.

The isometric imbeddability of $L_{1}(\rho) \otimes E$ into $W(\rho, E)$ was discovered independently by G. L. Seever whose work came to the attention of the present author through a personal communication.

Proof. From the remarks immediately preceding this theorem, it is plain that the assignment $\mathrm{t} \rightarrow m^{\mathrm{t}}$ effects an isomorphic imbedding of the algebraic tensor product $L_{1}(\rho) \otimes E$ into $W(\rho, E)$. We show that it is also isometric.

The assignment $t \rightarrow T^{t}$ is isometric by definition. One can see from the remarks preceding the formula (3.10) in $\S 3$ that $T^{\mathrm{t}}$ has the same norm as its restriction to $C(S)$. We denoted this restriction (with deliberate ambiguity) again by $T^{\mathrm{t}}: C(S) \rightarrow E$. In such a context $T^{\mathrm{t}}$ is the transformation assigned to $m^{t}$ under the imbedding (which is isometric) represented by the last inclusion in (3.12) (Theorem 3.1). It follows then that the assignments

$$
\mathrm{t} \rightarrow T^{\mathrm{t}} \rightarrow m^{\mathrm{t}}, \quad \text { for } \mathrm{t} \in L_{1}(\rho) \otimes E,
$$


are isometric, and that the isometry $\mathrm{t} \rightarrow m^{\mathrm{t}}$ can be extended to an isometry, indicated again by $\mathrm{t} \rightarrow m^{\mathrm{t}}$ of $L_{1}(\rho) \otimes_{\lambda} E$ into $W(\rho, E)$. As for relation (6.11), one can easily see that it reduces, for a finite tensor $\mathrm{t} \in L_{1}(\rho) \otimes E$ and $\psi \in C(S)$, to (3.6) with $m$ replaced by $m^{\mathrm{t}}$ and $T_{m}$ by $T^{\mathrm{t}}$ and to $(3.10)$ with $T_{f}$ replaced by $T^{\mathrm{t}}, f(s) d \rho(s)$ playing the role of $d m(s)$ and the $f$ being actually $f^{t}$. It follows that (6.11) is valid in this special case and that, because of its equivalent form (3.10), it can be extended to $\psi \in L_{\infty}(\rho)$. Now passing to the limit with respect to $t$, we obtain its validity for arbitrary

and $\psi \in L_{\infty}(\rho)$.

$$
t \in L_{1}(\rho) \otimes_{\lambda} E
$$

It remains to show that the isometry $\mathrm{t} \rightarrow m^{\mathrm{t}}$ maps $L_{1}(\rho) \otimes_{\lambda} E$ onto $W(\rho, E)$. This amounts to showing that every $m \in W(\rho, E)$ is of the form $m=m^{\text {t }}$ for $\mathrm{t} \in L_{1}(\rho) \otimes_{\lambda} E$. We do this. We have seen that $m$ defines a bounded linear transformation $T_{m}: L_{\infty}(\rho) \rightarrow E$. Let us first show that $T_{m}$ is a compact transformation. First, suppose that $m$ is of the form $m_{f}$ (see Theorem 3.1). Then $T_{m}$ is nothing but $T_{f}$, which we have seen is compact. On the other hand, since the assignments $f \rightarrow m_{f} \rightarrow T_{m_{f}}$ are isometric, and every $m \in W(\rho, E)$ is the limit in the semivariation norm of measures of the form $m_{f}, f$ Pettis integrable, it follows that $T_{m}$ is the uniform limit of compact linear transformations $T_{f}$ and hence compact.

Now that we have the compactness of $T_{m}$, we make use of a theorem of Grothendieck (12, p. 185, Proposition 41) which states that $L_{\infty}(\rho)$ satisfies the condition of metric approximation of Grothendieck. This implies (12, p. 164, Proposition 35), among other things, that every compact linear transformation of $L_{\infty}(\rho)$ into $E$ can be uniformly approximated by bounded linear transformations of finite rank. This actually boils down to the fact that $L_{1}(\rho) \otimes_{\lambda} E$ can be isometrically identified with the space of all compact linear transformations of $L_{\infty}(\rho)$ into $E$.

Let us now return to our $m$. We have that there exists a sequence of tensors $\mathrm{t}_{1}, \mathrm{t}_{2}, \ldots$ in $L_{1}(\rho) \otimes E$ such that $\left\|T_{m}-T^{\mathrm{t}_{n}}\right\| \rightarrow 0$ as $n$ tends to $\infty$. In particular $\mathrm{t}_{1}, \mathrm{t}_{2}, \ldots$ is a Cauchy sequence of $L_{1}(\rho) \otimes_{\lambda} E$ which converges to a tensor $\mathrm{t} \in L_{1}(\rho) \otimes_{\lambda} E$. Since $T_{m}$ must coincide with $T^{\mathrm{t}}$, it follows that $m=m^{\mathrm{t}}$. This completes our proof.

The next corollary follows immediately from Theorem 6.1 .

Corollary 6.1. Let $\rho$ be a non-negative, not necessarily finite measure on $S$. Then the space $W(\rho, E)$ can be identified isometrically with the space of all compact linear transformations of $L_{\infty}(\rho)$ into $E$. If $m \in W(\rho, E)$ and $T_{m}$ is the compact transformation that corresponds to $m$ under this identification, then $m$ and $T_{m}$ are related by the formula (6.11).

We now turn to another tensor product, the product $C^{\prime}(S) \otimes_{\gamma} E$ of the dual $C^{\prime}(S)$ of $C(S)$ and $E$, with respect to the greatest cross norm. This tensor product was the subject of a detailed study in (10), for $S$ compact. Our purpose 
here is not only to extend the study to locally compact spaces (which is more or less immediate) but to complete the earlier theory.

Now let $S$ be a general locally compact Hausdorff space. The general element $\mathrm{t}$ of $C^{\prime}(S) \otimes_{\gamma} E$ has a representation in terms of an absolutely convergent series of the form

$$
\mathrm{t}=\sum_{i=1}^{\infty} \mu_{i} \otimes x_{i}
$$

where $\mu_{i} \in C^{\prime}(S)$ and $x_{i} \in E . \gamma(\mathfrak{t})$ is given by

$$
\gamma(\mathrm{t})=\inf \sum_{i=1}^{\infty} \bar{\mu}_{i}(S)\left\|x_{i}\right\|
$$

where the infimum is taken over all representations (6.12) of $t$.

The proof of the following theorem will be omitted. It was given in (10, Theorem 4.2) for $S$ compact, and the extension to general locally compact $S$ is immediate. We continue to denote by $M(S, E)$ the Banach space of all measures $m: \mathbf{B}(S) \rightarrow E$ of finite variation with its total variation norm, and by $C^{\prime}(S)$ the dual space of $C(S)$.

THEOREM 6.2. There exists an isometric isomorphic imbedding represented by the inclusion

$$
C^{\prime}(S) \otimes_{\gamma} E \subset M(S, E) .
$$

If $\mathrm{t} \in C^{\prime}(S) \otimes_{\gamma} E$ has a representation (6.12) and $m_{\mathrm{t}}$ is the measure in $M(S, E)$ corresponding to $\mathbf{t}$ under the imbedding, then, for $A \in \mathbf{B}(S)$,

$$
m_{\mathfrak{t}}(A)=\sum_{i=1}^{\infty} \mu_{i}(A) x_{i} .
$$

The question of the possibility of actual equality in (6.14) was only partly considered in (10) where it was shown that there is equality if $E$ is a Phillips space. However, the general case was not discussed. It is our purpose here to complete the discussion. We show by an example that the inclusion (6.14) can be proper; then we shall proceed to give a necessary and sufficient condition in order for a measure $m \in M(S, E)$ to originate from $C^{\prime}(S) \otimes_{\gamma} E$. We finally show that the validity of an equality in (6.14) actually characterizes Phillips spaces. We begin with an example of a measure $m \in M(S, E)$, which does not belong to $C^{\prime}(S) \otimes_{\gamma} E$. The following construction is an adaptation to the present purposes of an example due to Grothendieck (12, p. 128, top).

We use for $E$ the space $C^{\prime}(S)$ and exhibit an element $m \in M\left[S, C^{\prime}(S)\right]$ not belonging to $C^{\prime}(S) \otimes_{\gamma} C^{\prime}(S)$. Now, by the theorem of Ivan Singer (20) quoted above (see $\$ 4$, in particular (4.4)), we have the first of the following two equalities:

$$
M\left[S, C^{\prime}(S)\right]=C^{\prime}[S, C(S)]=C^{\prime}(S \times S) .
$$


The second equality follows from the familiar fact that $C[S, C(S)]$ can be identified naturally with $C(S \times S)$. The equalities in (6.16) represent isometric isomorphisms. Hence, our example can be given as an $m \in C^{\prime}(S \times S)$, a bounded linear functional on $C(S \times S)$. For that purpose, we specialize our $S$ to be the interval $[0,1]$. Now the functional is defined, for $f \in C(S \times S)$, by

$$
\langle f, m\rangle=\int_{0}^{1} f(s, s) d s .
$$

Now $m$ as a vector measure $m: B(S) \rightarrow C^{\prime}(S)$ defines a bounded linear transformation $T_{m}: S(S) \rightarrow C^{\prime}(S)$, given, for instance, by (3.6), for $E^{\prime}=C^{\prime}(S)$. On the other hand, it is known that if $m$ were an element of $C^{\prime}(S) \otimes_{\gamma} C^{\prime}(S)$, under the inclusion (6.14), then $T_{m}$, given by a formula of the type (6.6), would be compact. Consequently, to show that $m$ provides the desired example, it suffices to show that $T_{m}$ is not compact. To do this we compute $T_{m} \psi$ for $\psi \in C(S)$. One sees easily, for $\phi \in C(S)$, that

$$
\left\langle\phi, T_{m} \psi\right\rangle=\int_{0}^{1} \phi(s) \psi(s) d s .
$$

Hence $T_{m} \psi$ is the measure on $[0,1]$ with $\psi$ as Radon-Nikodym derivative. From this, it follows immediately that the proof that $T_{m}$ is not compact boils down to showing that the solid unit sphere (with respect to \|\|$_{\infty}$ ) of $C([0,1])$ is not totally bounded with respect to \|\|$_{1}$, a well-known fact, verified easily by finding an infinite uniformly bounded sequence of continuous functions on $[0,1]$, any two of which are at a \|\|$_{1}$-distance of $1 / 2$ apart. This completes our discussion of our example of an $m \in M(S, E)$ which does not originate from $C^{\prime}(S) \otimes_{\gamma} E$ in (6.14).

We next give a theorem characterizing those measures of $M(S, E)$ that belong to $C^{\prime}(S) \otimes_{\gamma} E$, under the imbedding (6.14).

Theorem 6.3. Let $m$ belong to $M(S, E)$. Then $m$ belongs to $C^{\prime}(S) \otimes_{\gamma} E$ under the imbedding represented by (6.14) if and only if it has a strong Radon-Nikodym derivative with respect to $\mu^{m}$; i.e., if and only if there exists a strongly $\mu^{m}$-measurable mapping $h_{m}: S \rightarrow E$ such that $\left\|h_{m}(s)\right\|=1 \mu^{m}$-almost everywhere and such that, for every $A \in \mathbf{B}(S)$, we have

$$
m(A)=\int_{A} h_{m}(s) d \mu^{m} .
$$

Proof. Suppose first that such a mapping $h_{m}: S \rightarrow E$ exists. We show that $m \in C^{\prime}(S)$. Now since $h_{m}$ is essentially bounded and strongly measurable with respect to the finite measure $\mu^{m}$, it follows that $h_{m} \in L_{1}\left(\mu^{m}, E\right)$. We now make use of a theorem of Grothendieck (12, Theorem 2, p. 59) which states that

$$
L_{1}\left(\mu^{m}, E\right)=L_{1}\left(\mu^{m}\right) \otimes_{\gamma} E .
$$


It follows that, for $s \in S$,

$$
h_{m}(s)=\sum_{i=1}^{\infty} \phi_{i}(s) x_{i},
$$

$\phi_{i} \in L_{1}\left(\mu^{m}\right)$ and $x_{i} \in E$ with

$$
\sum_{i=1}^{\infty}\left\|\phi_{i}\right\|_{1}\left\|x_{i}\right\|<+\infty .
$$

Furthermore, it follows from the same theorem that

$$
\left\|h_{m}\right\|_{1}=\inf \sum_{i=1}^{\infty}\left\|\phi_{i}\right\|_{1}\left\|x_{i}\right\|
$$

where the infimum is taken over all the expression to the left of (6.21) as we consider all possible representations of $h_{m}$ of the form (6.20). Let now $\mu_{i}$ be the scalar measure whose Radon-Nikodym derivative is $\phi_{i}$. We easily verify that

$$
m=\sum_{i=1}^{\infty} \mu_{i} \otimes x_{i}
$$

and that (6.23) represents a tensor in $C^{\prime}(S) \otimes_{\gamma} E$ as was claimed.

Conversely, suppose that $m$ originates from a tensor from $C^{\prime}(S) \otimes_{\gamma} E$. Then it has a representation (6.23) with

$$
\sum_{i=1}^{\infty} \bar{\mu}_{i}(S)\left\|x_{i}\right\|<+\infty .
$$

It is easy to find a regular measure $\nu$, with respect to which each $\mu_{i}$ is absolutely continuous. We can put, for instance

$$
\nu=\sum_{i=1}^{\infty} \bar{\mu}_{i} / 2^{i}\left[1+\bar{\mu}_{i}(S)\right] ;
$$

see $(13$, p. 371$)$. Let $\omega_{i}$ be the Radon-Nikodym derivative of $\mu_{i}$ with respect to $\nu$ and

$$
f(s)=\sum_{i=1}^{\infty} \omega_{i}(s) x_{i},
$$

for $s \in S$. It is easy to see that $f \in L_{1}(\nu, E)$ and that $m$ and $f$ are related in the same way that $m_{f}$ and $f$ are related in (5.2). The desired representation of $m$ now follows from Theorem 5.1. This completes our proof.

Theorem 6.4. A Banach space $E$ is a Phillips space if and only if, for every locally compact Hausdorff space $S$,

$$
C^{\prime}(S) \otimes_{\gamma} E=M(S, E) .
$$

Proof. Suppose that $E$ is a Phillips space. Then, for $S$ compact, the equation (6.26) is the essential content of (10, Theorem 4.4). We have simply to extend the result to locally compact spaces. We do this with the aid of the one-point 
compactification $S_{\infty}$. Let $m \in M(S, E)$. By Corollary $3.1, m$ can be extended to a measure $m_{\infty}$ on $S_{\infty}$ with values in $E$, which is concentrated on $S \subset S_{\infty}$. One can easily show that the corresponding extension of $\mu^{m}$ is the variation measure of $m_{\infty}$; hence the latter is of finite variation and belongs to $M\left(S_{\infty}, E\right)$, from which it follows, since $E$ is a Phillips space, that $m_{\infty}$ originates from a tensor in $C^{\prime}\left(S_{\infty}\right) \otimes_{\gamma} E$. Therefore (Theorem 6.3) $m_{\infty}$ has a representation of the form (5.1). Let us denote by $h_{\infty}$ the $h_{m}$ appearing in (5.1) for $m_{\infty}$. One shows easily that $h_{\infty}$ vanishes almost (with respect to $\mu^{m}$ ) everywhere outside of $S \subset S_{\infty}$. It follows that $m$ itself has a representation (5.1) and, by Theorems 5.1 and 6.3 , that $m$ originates from a tensor in $C^{\prime}(S) \otimes_{\gamma} E$.

Suppose conversely that $(6.26)$ is valid for every locally compact Hausdorff space $S$. We show that $E$ is a Phillips space. For the definition of this concept we refer the reader to $\S 5$. Let $S$ be a compact Hausdorff space, $\mu$ a non-negative measure on $S$, and $T: L_{1}(\mu) \rightarrow E$ a bounded linear transformation. Since $\mu$ is finite, $L_{\infty}(\mu) \subset L_{1}(\mu)$, and the following definition has a meaning

$$
m(A)=T \chi_{A},
$$

where $\chi_{A}$ denotes the characteristic function of a Borel subset $A$ of $S$. Clearly (6.27) defines a strong measure $m: \mathbf{B}(S) \rightarrow E$ and $\|m(A)\| \leqslant\|T\| \mu(A)$. From here it follows that $m$ has finite variation, i.e., $m \in M(S, E)$. We then have, by hypothesis, that $m$ originates from a tensor in $C^{\prime}(S) \otimes_{\gamma} E$, and, by Theorem 6.3 , that $m$ has a representation (6.19) where $h_{m}$ is strongly $\mu^{m}$ measurable and $\left\|h_{m}(s)\right\|=1, \mu^{m}$-almost everywhere. Now $\mu^{m}$ is absolutely continuous with respect to $\mu$. Let $\theta$ be its Radon-Nikodym derivative with respect to $\mu$. Then from the representation (6.19) and manipulations involving the ordinary Radon-Nikodym theorem for scalar-valued functions, we obtain, at least formally, the relation

$$
m(A)=\int_{A} h_{m}(s) \theta(s) d \mu(s)
$$

for $A \in \mathbf{B}(S)$. However, in order to give (6.28) a strict meaning, and indeed to complete our proof, we must show that the integrand $h_{m}(s) \theta(s)$ is strongly $\mu$-measurable, or that it can be replaced by an equivalent integrand that is $\mu$-measurable (the procedure chosen by us). Since the image $h_{m}(P)$ is separable, for some subset $P$ of $S$ with $\mu^{m}$-null complement, we may replace $h_{m}$ in (6.28) by $h_{m} \chi_{P}$. This does not change the integral on the right of (6.28), and the new mapping has separable image and is strongly measurable with respect to $\mu^{m}$. Hence, we may assume that $h_{m}$ has separable image $h_{m}(S)$. It follows then, from (8, Theorem 4, p. 179), that $h_{m}(s) \theta(s)$ is weakly $\mu$-measurable. This and the separability of the image yield, again by the theorem of Pettis in (18), that $h_{m}(s) \theta(s)$ is strongly $\mu$-measurable. If we set $h(s)=h_{m}(s) \theta(s)$, this gives the desired representation of $T$ in the definition of a Phillips space. This completes our proof. 
Let us now specialize our considerations to transformation-valued measures $m: \mathbf{B}(S) \rightarrow \mathbf{L}\left(E, F^{\prime}\right)$, namely vector-valued measures, in the sense of $\$ 3$, but where the general image vector space $E$ has been replaced by the space $\mathbf{L}\left(E, F^{\prime}\right)$ of all bounded linear transformations of $E$ into the dual space $F^{\prime}$ of a Banach space $F$. We begin with some notational adjustments necessitated by the complicated character of some of the expressions soon to be considered. For $U \in \mathbf{L}\left(E, F^{\prime}\right)$, the usual notation $U x$ for the image of $x \in E$ under $U$ will be used along with the notation $\{x, U\}$, which is more convenient and suggestive in many cases. Until now, the integral

$$
\int_{S} \phi(s) d m(s) \quad \text { for } \phi \in L_{1}\left(\mu^{m}\right)
$$

has sufficed for our discussion of general vector measures of finite variation. For transformation measures of finite variation, another integral, denoted by

$$
\int_{S}\{f(s), d m(s)\} \quad \text { for } f \in L_{1}\left(\mu^{m}, E\right)
$$

becomes important. The special notation is our own, but the concept is introduced and explained in (5), to which we refer the reader for details. For simple mappings

$$
f(s)=\sum_{i=1}^{n} \chi_{A i}(s) x_{i}, \quad A_{i} \in B(S) \text { and } x_{i} \in E,
$$

$\int_{s}\{f(s), d m(s)\}$ is defined by

$$
\int_{S}\{f(s), d m(s)\}=\sum_{i=1}^{n}\left\{x_{i}, m\left(A_{i}\right)\right\} .
$$

The definition is then extended by continuity to the entire $L_{1}\left(\mu^{m}, E\right)$ by virtue of the fact that the mappings of the form (6.29) are dense in $L_{1}\left(\mu^{m}, E\right)$. We shall make free use of the basic properties of this new integral, without further discussion. In particular, if $F$ is the field of scalars, $\mathbf{L}\left(E, F^{\prime}\right)$ becomes simply $E^{\prime}$ and we use the more suggestive notation

$$
\int_{s}\langle f(s), d m(s)\rangle \text {. }
$$

For $F$ separable, Dinculeanu and Foias (5, Theorem 2, p. 537) have given a Radon-Nikodym representation of the type (6.19) for transformation measures. Although the measurability properties of the Radon-Nikodym derivative involved are somewhat weaker than those considered above in connection with Theorems 6.3 and 6.4, their representation has the virtue of being valid for all transformations measures $m: \mathbf{B}(S) \rightarrow \mathbf{L}\left(E, F^{\prime}\right)$, and not just for those measures originating from the appropriate tensor product. On the other hand their proof is quite long. We are going to give here a new proof (Theorem 6.5, below) of the theorem of Dinculeanu and Foias, based on the theory of topological tensor products. The present proof is much shorter than 
the original one. It also enables us to remove the assumption, made in the original theorem, that $F$ is separable, by applying a general version of the Dunford-Pettis theorem due to A. and C. Ionescu Tulcea (16). We are indebted to the referee of (10) for having pointed out the possibility of extending our proof beyond the realm of separable spaces.

Theorem 6.5 (Dinculeanu-Foias). Let $m: \mathbf{B}(S) \rightarrow \mathbf{L}\left(E, F^{\prime}\right)$ be a measure of finite variation. Then there exists a mapping $f: S \rightarrow \mathbf{L}\left(E, F^{\prime}\right)$ with the following properties:

(1) $\|f(s)\|=1$, for $\mu^{m}$-almost every $s \in S$.

(2) For every $x \in E$, the mapping $f^{x}: S \rightarrow F^{\prime}$, defined by $f^{x}(s)=\{x, f(s)\}$, is weakly $\mu^{m}$-measurable.

(3) For every $y \in F$ and $g \in L_{1}\left(\mu^{m}, E\right)$,

$$
\left\langle y, \int_{S}\{g(s), d m(s)\}\right\rangle=\int_{S}\langle y,\{g(s), f(s)\}\rangle d \mu^{m}(s) .
$$

Proof. For $\phi \in L_{1}\left(\mu^{m}\right)$, the formula

$$
W \phi=\int_{S} \phi(s) d m(s)
$$

defines a bounded linear transformation of $L_{1}\left(\mu^{m}\right)$ into $\mathbf{L}\left(E, F^{\prime}\right)$. Clearly $\|W\| \leqslant 1$. We know from $(6.5)$ that $\mathbf{L}\left(E, F^{\prime}\right)$ is nothing but the dual space of $E \otimes_{\gamma} F$. By the generalized form of the Dunford-Pettis theorem due to A. and C. Ionescu Tulcea (16, Section 4, p. 189), we conclude that there exists a weakly* measurable mapping

so that

$$
f: S \rightarrow \mathbf{L}\left(E, F^{\prime}\right)=\left(E \otimes_{\gamma} F\right)^{\prime}
$$

and

$$
\underset{s \in S}{\operatorname{ess} \sup _{S}}\|f(s)\|=\|W\| \leqslant 1
$$

$$
\langle\mathrm{t}, W\rangle=\int_{S}\langle\mathrm{t}, f(s)\rangle \phi(s) d \mu^{m}(s)
$$

for every $\mathrm{t} \in E \otimes_{\gamma} F$ and $\phi \in L_{1}\left(\mu^{m}\right)$. If we specialize (6.33) to tensors $\mathrm{t}=x \otimes y$ for $x \in E$ and $y \in F$, we obtain

$$
\left\langle y,\left\{x, \int_{S} \phi(s) d m\right\}\right\rangle=\int_{S}\langle y,\{x, \phi(s) f(s)\}\rangle d \mu^{m}(s) .
$$

Now, for fixed $y \in F$, each side of relation (6.31) represents a bounded linear functional on the space $L_{1}\left(\mu^{m}, E\right)$, as we let $g$ vary over $L_{1}\left(\mu^{m}, E\right)$. Now the relation (6.34) is merely the statements that these two functionals agree on the subset $P$ of $L_{1}\left(\mu^{m}, E\right)$ consisting of mappings $g$ of the form $g(s)=\phi(s) x$, for $x \in E$, and $\phi \in L_{1}\left(\mu^{m}\right)$. Now $P$ is total in $L_{1}\left(\mu^{m}, E\right)$. This follows, for example, from a theorem of Grothendieck (12, Theorem 2, p. 59). We conclude that (6.31) is valid for arbitrary $g \in L_{1}\left(\mu^{m}, E\right)$. We see easily that the weak* measurability 
of $f: S \rightarrow\left(E \otimes_{\gamma} F\right)^{\prime}$ implies the weak* $\mu^{m}$-measurability of $f^{x}$, for every $x$, and much more, since the latter measurability is clearly equivalent to the $\mu^{m}$ measurability of every scalar-valued function of the form

$$
\langle x \otimes y, f(s)\rangle=\langle y,\{x, f(s)\}\rangle .
$$

We now establish Property (1) of our theorem. Suppose that $f$ does not have this property. Then for some $\epsilon<1$, there exists a set $A \in \mathbf{B}(S)$ such that $\mu^{m}(A)>0$ and $\|f(s)\|<\epsilon$, for $s \in A$. On the other hand, for any disjoint sets $A_{1}, A_{2}, \ldots, A_{n} \in \mathbf{B}(S)$, such that

we have

$$
A=\bigcup_{i=1}^{n} A_{i}
$$

$$
\begin{aligned}
\sum_{i=1}^{n}\left\|m\left(A_{i}\right)\right\| & =\sum_{i=1}^{n}\left\|\int_{S} \chi_{A i}(s) d m(s)\right\| \\
& =\sum_{i=1}^{n}\left\|\int_{A_{i}} f(s) d \mu^{m}(s)\right\| .
\end{aligned}
$$

We have also, for every $i, x \in E$, and $y \in F$,

$$
\begin{aligned}
\left\|\int_{S} \chi_{A i}(s) d m(s)\right\| & =\sup \left|\left\langle y, \int_{S}\left\{x \chi_{A i}(s), d m(s)\right\}\right\rangle\right| \\
& =\sup \mid\left\langle y, \int_{A_{i}}\left\{x, f(s)\left\{d \mu^{m}(s)\right\rangle \mid \leqslant \epsilon \mu^{m}\left(A_{i}\right) .\right.\right.
\end{aligned}
$$

In (6.36) the supremum is always taken as $x$ and $y$ range over the respective unit spheres of $E$ and $F$. It follows from (6.35) and (6.36) and the definition of $\mu^{m}$ that $\mu^{m}(A) \leqslant \epsilon \mu^{m}(A)<\mu^{m}(A)$, a contradiction. The essential uniqueness of $f$ follows from the corresponding clause in the generalized Dunford-Pettis theorem. This concludes our proof.

We now consider one final question which is best discussed in the light of the theory of tensor products. The concept of weak* vector measure, contrasted with that of strong vector measure due to Bartle, Dunford, and Schwartz (1), was introduced in (10) and discussed in $\$ 3$ of the present work. To bring out some of the interest of weak* measures, we gave in $\$ 3$ an example of one such measure that is not a strong vector measure. However, this example is somewhat artificial. Now with the help of the theory of tensor products we can show that the spectral resolutions of the identity, in the sense of Dunford (7), associated with many spectral operators, are vector (specifically, operator) valued measures, whose values lie in dual spaces of Banach spaces and that such measures are weak* vector measures, which are not strong vector measures. For the details of the theory of spectral operators, of which we use only the most elementary facts, we refer the reader to Dunford's address (7). 
We shall now consider operators on the dual space $E^{\prime}$ of a Banach space $E$. This, of course, includes operators on reflexive spaces, as well as many others. The spectral resolution of an operator $T \in \mathbf{L}\left(E^{\prime}\right)$, the algebra of all bounded operators on $E^{\prime}$, is (among other things) a projection-valued mapping $p: \mathbf{B}(S) \rightarrow \mathbf{L}\left(E^{\prime}\right)$ ( $S$ in this context stands for the complex plane), which is countably additive with respect to the strong operator topology of $\mathbf{L}\left(E^{\prime}\right)$. We spell out the meaning of this type of countable additivity in the form in which we shall use it here. It means that, for every increasing sequence $A_{1} \subset A_{2} \subset \ldots$ of Borel sets in the plane and every $x^{\prime} \in E^{\prime}$, we have

$$
p\left(\bigcup_{i=1}^{\infty} A_{i}\right) x^{\prime}=\lim _{i \rightarrow \infty} p\left(A_{i}\right) x^{\prime}
$$

where the limit is taken with respect to the strong topology of $E^{\prime}$. Now, by (6.5), the space $\mathbf{L}\left(E^{\prime}\right)$, with its usual norm, is the dual space of the tensor product $E \otimes_{\gamma} E^{\prime}$. We are going to show that

$$
p: \mathbf{B}(S) \rightarrow \mathbf{L}\left(E^{\prime}\right)=\left(E \otimes_{\gamma} E^{\prime}\right)^{\prime}
$$

is a weak* vector measure. Now the duality between $E \otimes_{\gamma} E^{\prime}$ and $\mathbf{L}\left(E^{\prime}\right)$ is implemented in the following manner. Every $\mathrm{t} \in E \otimes_{\gamma} E^{\prime}$ has a representation (see $(6.3))$ of the form

$$
\mathrm{t}=\sum_{i=1}^{\infty} x_{i} \otimes x^{\prime}{ }_{i}
$$

where

$$
\sum_{i=1}^{\infty}\left\|x_{i}\right\|\left\|x_{i}^{\prime}\right\|<+\infty .
$$

We now have, for every $U \in \mathbf{L}\left(E^{\prime}\right)$,

$$
\langle\mathrm{t}, U\rangle=\sum_{i=1}^{\infty}\left\langle x_{i}, U x^{\prime}{ }_{i}\right\rangle .
$$

THEOREM 6.6. Let $S$ stand for the complex plane. Let $p: \mathbf{B}(S) \rightarrow \mathbf{L}\left(E^{\prime}\right)$ be the spectral resolution of a spectral operator on $E^{\prime}$. Then $p$ is a weak* vector measure.

Proof. We refer the reader to $\S 3$ for the definition of weak* vector measures. Let $\mathrm{t} \in E \otimes_{\gamma} E^{\prime}$. One must prove that the scalar-valued set function $\mu_{\mathrm{t}}$ (this notation agrees with the notation $\mu_{x}$ of $\S 3$ ) is a scalar measure on $S$. The crux of the matter is showing that $\mu_{\mathrm{t}}$ is countably additive. Let the Borel set $A \subset S$ be the union of an increasing sequence $A_{1} \subset A_{2} \subset \ldots$ of Borel sets. We show that $\mu_{\mathrm{t}}(A)=\lim _{k} \mu_{\mathrm{t}}\left(A_{k}\right)$.

Let $\epsilon>0$. We know that there exists $\kappa>0$ such that $\|p(B)\|<\kappa$ for every Borel set $B \subset S$. Let $\mathrm{t}$ have a representation (6.38) and $n$ be a positive integer such that

$$
\sum_{i>n}\left\|x_{i}\right\|\left\|x_{i}^{\prime}\right\|<\epsilon / \kappa
$$


Now, since $p$ is countably additive with respect to the strong operator topology, there exists a $k_{0}$ such that

$$
\sum_{i=1}^{n}\left|\left\langle x_{i}, p(A) x^{\prime}{ }_{i}-p\left(A_{k}\right) x^{\prime}{ }_{i}\right\rangle\right|<\epsilon
$$

for every $k>k_{0}$. Hence, for every such $k$, using (6.41), we have

$$
\begin{aligned}
\left|\mu_{t}(A)-\mu_{t}\left(A_{k}\right)\right| & \leqslant \sum_{i=1}^{n}\left|\left\langle x_{i}, p(A) x_{i}^{\prime}-p\left(A_{k}\right) x^{\prime}{ }_{i}\right\rangle\right| \\
& +\|p(A)\| \sum_{i>n}\left\|x_{i}\right\|\left\|x_{i}^{\prime}\right\|+\left\|p\left(A_{k}\right)\right\| \sum_{i>n}\left\|x_{i}\right\|\left\|x_{i}^{\prime}\right\| .
\end{aligned}
$$

This completes the proof of the countable additivity of $\mu_{\mathrm{t}}$. It remains to show that $\mu_{\mathrm{t}}$ is regular. However, this follows from the well-known fact (14, Theorem G, p. 228) that every scalar-valued (Borel) measure on the plane is regular. This completes our proof.

As is well known, spectral resolutions of operators are seldom strong measures (in our nomenclature) for this would mean countable additivity with respect to the uniform topology of $\mathbf{L}\left(E^{\prime}\right)$, a property absent even in the most elementary instances of spectral resolutions on infinite-dimensional spaces, such as those of hermitean compact operators in Hilbert spaces. Thus Theorem 6.6 provides interesting examples of weak* vector measures that are not strong vector measures.

Note. The author is grateful to G. L. Seever for calling his attention to the proof of Pettis (18, p. 303) of the fact that the space of all Pettis integrable mappings of the unit interval (with Lebesgue measure) into an infinitedimensional Hilbert space is not complete. This answers in the negative a question raised in the present paper in the paragraph immediately preceding Theorem 3.2.

\section{REFERENCES}

1. R. G. Bartle, N. Dunford, and J. Schwartz, Weak compactness and vector measures, Can. J. Math., 7 (1955), 288-305.

2. N. Dinculeanu, Mesures vectorielles et opérations linéaires, C. R. Acad. Sci. Paris, 246 (1958), $2327-2331$.

3. - Représentation intégrale d'opérations linéaires III, Proc. Amer. Math. Soc., 10 (1959), $59-68$.

4. N. Dinculeanu et C. Foias, Mesures vectorielles et opérations linéaires sur $L_{E}{ }^{p}, \mathrm{C}$. R. Acad. Sci. Paris, 248 (1959), 1759-1762

5. - Sur la représentation intégrale de certains opérateurs linéaires IV. Opérateurs linéaires sur les espaces $L_{\mathfrak{a}}{ }^{p}$, Can. J. Math., 13 (1961), 529-556.

6. N. Dunford, Integration and linear operations, Trans. Amer. Math. Soc., 40 (1936), 474-494.

7. - A survey of the theory of spectral operators, Bull. Amer. Math. Soc., 64 (1958), 217-274.

8. N. Dunford and J. Schwartz, Linear operators, Part I (New York, 1958).

9. I. Gelfand, Abstrakte Funktionen und lineare Operatoren, Rec. Math. (Mat. Sb.), NF, 4 (1938), 238-284. 
10. J. Gil de Lamadrid, Measures and tensors, Trans. Amer. Math. Soc., 114 (1965), 98-121.

11. A. Grothendieck, Sur les applications linéaires faiblement compact d'espaces de type $C(K)$, Can. J. Math., 5 (1953), 129-173.

12. - Produits tensoriels topologiques et espaces nucléaires, Mem. Amer. Math. Soc., 16 (1955).

13. - La théorie de Fredholm, Bull. Soc. Math. France, 84 (1956), 319-384.

14. P. R. Halmos, Measure theory (Princeton, 1950).

15. E. Hille and R. S. Phillips, Functional analysis and semi-groups (Providence, 1957).

16. A. and C. Ionescu Tulcea, On the lifting property II. Representation of linear operators on spaces $L_{E}{ }^{r}, 1 \leqslant r<\infty$, J. Math. Mech., 11 (1962), 113-195.

17. S. Kakutani, Concrete representations of abstract $(M)$ spaces ( $A$ characterization of the space of continuous functions), Ann. Math. (2), 42 (1941), 994-1024.

18. B. J. Pettis, On integration in vector spaces, Trans. Amer. Math. Soc., 44 (1938), 277-304.

19. R. Schatten, $A$ theory of cross spaces, Ann. Math. Studies, 26 (Princeton, 1955).

20. I. Singer, Linear functionals on spaces of continuous mappings of a compact Hausdorff space into a Banach space (Russian), Rev. Math. Pures Appl., 2 (1957), 309-314.

University of Minnesota, Minneapolis, Minnesota, and

Centre Universitaire International, Paris 